KEN YAMAMURA

\title{
Maximal unramified extensions of imaginary quadratic number fields of small conductors
}

Journal de Théorie des Nombres de Bordeaux, tome 9, no 2 (1997), p. $405-448$

<http://www.numdam.org/item?id=JTNB_1997_9_2_405_0>

(C) Université Bordeaux 1, 1997, tous droits réservés.

L'accès aux archives de la revue «Journal de Théorie des Nombres de Bordeaux » (http://jtnb.cedram.org/) implique l'accord avec les conditions générales d'utilisation (http://www.numdam.org/conditions). Toute utilisation commerciale ou impression systématique est constitutive d'une infraction pénale. Toute copie ou impression de ce fichier doit contenir la présente mention de copyright.

\section{Numdam}

Article numérisé dans le cadre du programme

Numérisation de documents anciens mathématiques

http://www.numdam.org/ 
Journal de Théorie des Nombres

de Bordeaux 9 (1997), 405-448

\title{
Maximal unramified extensions of imaginary quadratic number fields of small conductors
}

\author{
par KEN YAMAMURA
}

\begin{abstract}
RÉSUMÉ. Nous déterminons la structure du groupe de Galois $\operatorname{Gal}\left(K_{u r} / K\right)$ de l'extension maximale non ramifiée $K_{u r}$ de chaque corps quadratique imaginaire de conducteur $\leqq 420$ ( l'extension $K_{u r}$ coïncide avec $K$, ou avec le corps de classes de Hilbert de $K$, ou avec le second corps de classes de Hilbert de $K$ ou avec le troisième corps de classes de Hilbert de $K$. Les bornes d'Odlyzko sur les discriminants et les informations sur la structure des groupes de classes obtenues par l'action du groupe de Galois sur les groupes de classes sont ici essentielles. Nous utilisons aussi des relations sur le nombre de classes et un ordinateur pour le calcul du nombre de classes de corps de bas degré pour obtenir le nombre de classes de corps de degré plus élevé. Nous utilisons aussi des résultats sur les tours de corps de classes, ainsi que notre connaissance des 2-groupes d'ordre $\leqq 2^{6}$ et des groupes linéaires sur des corps finis.
\end{abstract}

ABSTRACT. We determine the structures of the Galois groups $\mathrm{Gal}\left(K_{u r} / K\right)$ of the maximal unramified extensions $K_{u r}$ of imaginary quadratic number fields $K$ of conductors $\leqq 420(\leqq 719$ under the Generalized Riemann $\mathrm{Hy}$ pothesis). For all such $K, K_{u r}$ is $K$, the Hilbert class field of $K$, the second Hilbert class field of $K$, or the third Hilbert class field of $K$. The use of Odlyzko's discriminant bounds and information on the structure of class groups obtained by using the action of Galois groups on class groups is essential. We also use class number relations and a computer for calculation of class numbers of fields of low degrees in order to get class numbers of fields of higher degrees. Results on class field towers and the knowledge of the 2-groups of orders $\leqq 2^{6}$ and linear groups over finite fields are also used.

\section{INTRODUCTION}

Let $K$ be an algebraic number field (of finite degree) and $K_{u r}$ its maximal unramified extension. Then the Galois group $\operatorname{Gal}\left(K_{u r} / K\right)$ can be

1991 Mathematics Subject Classification. Primary 11R32,11R11.

Key words and phrases. Maximal unramified extension, imaginary quadratic number field, discriminant bounds, class field tower.

Manuscrit reçu le 17 avril 1997 
both finite and infinite and in general it is quite difficult to determine the structure of this group. If $K$ has sufficiently small root discriminant, then $K_{u r}=K$, that is, $K$ has no nontrivial unramified extension. This is the case, for example, for the imaginary quadratic number fields with class number one, the cyclotomic number fields with class number one, the real abelian number fields of prime power conductors $\leqq 67$ (see [57, Appendix]). For some fields $K$ with small root discriminant, we can determine $\operatorname{Gal}\left(K_{u r} / K\right)$. The purpose of this paper is to determine the structure of $\mathrm{Gal}\left(K_{u r} / K\right)$ of imaginary quadratic number fields $K$ of small conductors. For imaginary quadratic number fields $K$ of conductors $\leqq 420(\leqq 719$ under the Generalized Riemann Hypothesis $(\mathrm{GRH}))$ we determine $\mathrm{Gal}\left(K_{u r} / K\right)$ and tabulate them for $K$ with $K_{u r} \neq K_{1}$, where $K_{1}$ denotes the Hilbert class field of $K$. (If $K_{u r}=K_{1}$, then $\operatorname{Gal}\left(K_{u r} / K\right)=\operatorname{Gal}\left(K_{1} / K\right) \cong \mathrm{Cl}(K)$, the class group of $K$ by class field theory.) For all such $K, K_{u r}$ is one of $K, K_{1}, K_{2}$, or $K_{3}$, where $K_{2}$ (resp. $K_{3}$ ) is the second (resp. third) Hilbert class field of $K$. In other words, $K_{u r}$ coincides with the top of the class field tower of $K$ and the length of the tower is at most three. If possible, we give also simple expressions of $K_{1}$ and $K_{2}$. Also for $K=\mathrm{Q}(\sqrt{d})$ with $723 \leqq|d|<1000$, we tabulate $\operatorname{Gal}\left(K_{u r} / K\right)$ except for some $d$.

From now on let $K=\mathbf{Q}(\sqrt{d})$ be an imaginary quadratic number field with discriminant $d<0$. J. Martinet stated in [34] that if $|d|<250$, then $K_{u r}=K_{1}$ except for 7 fields, for which he gave the structure of $\operatorname{Gal}\left(K_{u r} / K\right)$. (We note that $\operatorname{Gal}\left(K_{u r} / K\right) \cong H_{24}$ for $K=\mathbf{Q}(\sqrt{-248})$ in [34] is false ${ }^{1}$.) He also stated that this fact is proved by using the methods which J. Masley [35] (and later F. J. van der Linden [50]) used for calculation of class numbers of real abelian number fields of small conductors. They used Odlyzko's discriminant bounds and information on the structure of class groups obtained by using the action of Galois groups on class groups. In addition to their methods, we use a computer for calculation of class numbers of fields of low degrees (we use KANT) and then use class number relations to get class numbers of fields of higher degrees (see §3). Results on class field towers $[5,25,29,31,49]$ and the knowledge of the 2-groups of orders $\leqq 2^{6}[17]$ and linear groups over finite fields (see $\S 4$ ) are also used.

We know that if $|d| \leqq 499(|d| \leqq 2003$ under GRH), then the degree $\left[K_{u r}: K\right]$ is finite (see $\left.\S 2\right)$. For these $d$, we want to determine $\operatorname{Gal}\left(K_{u r} / K\right)$. The key fact is that any unramified (finite) extension $L$ of $K$ has the same root discriminant as $K: r d_{L}=\left|d_{L}\right|^{1 /[L: Q]}=r d_{K}=\sqrt{|d|}$. Thus, if we have $r d_{K}<B(2 N)$, where $B(2 N)$ denotes the lower bound for the root

\footnotetext{
${ }^{1}$ The referee pointed out that this was corrected by Martinet in a supplement to his paper which he distributed along with the original reprint.
} 
discriminants of the totally imaginary number fields of (finite) degrees $\geqq$ $2 N$, then we get $\left[K_{u r}: K\right]<N$. We do not know the real values of $B(2 N)$ (except for $N \leqq 4)$, however, some lower bounds for $B(2 N)$ are known. The best known unconditional lower bounds for $B(2 N)$ can be found in the tables due to F. Diaz y Diaz [12]. If we assume the truth of GRH, much better lower bounds can be obtained. The best known conditional (GRH) lower bounds are found in the unpublished tables due to A. M. Odlyzko [38], which are copied in Martinet's expository paper [34]. Let $K_{l}$ be the top of the class field tower of $K: K=K_{0} \subseteq K_{1} \subseteq K_{2} \subseteq \cdots$ $\left(K_{i+1}\right.$ is the Hilbert class field of $\left.K_{i}\right)$, that is, $l$ is the smallest number with $K_{l+1}=K_{l}$. If we cannot get $\left[K_{u r}: K_{l}\right]<60$, which implies $K_{u r}=K_{l}$, from available lower bounds for $B(2 N)$, we need to judge whether $K_{l}$ has an unramified nonsolvable Galois extension and this is quite difficult. For the fields $\mathbf{Q}(\sqrt{-423})$ and $\mathbf{Q}(\sqrt{-723})$, we have $h\left(K_{1}\right)=1$, that is, $l=1$ and we cannot get $\left[K_{u r}: K_{1}\right]<60$ from available lower bounds for $B(2 N)$ (even under GRH for $\mathrm{Q}(\sqrt{-723})$ ). For $|d| \leqq 420(|d| \leqq 719$ under GRH), we get $\left[K_{u r}: K_{l}\right]<60$ and our main problem is to determine the degree $\left[K_{l}: \mathrm{Q}\right]$. In general, it is difficult to determine $\left[K_{2}: \mathrm{Q}\right]$, because it is very hard to calculate the class number $h\left(K_{1}\right)$ of $K_{1}$. (Of course, for $K$ with small $\mathrm{Cl}(K)$, we can calculate $h\left(K_{1}\right)$ with the help of a computer.) Now let $K_{g}$ be the genus field of $K$, that is, the maximal unramified abelian extension of $K$ which is abelian over $\mathbf{Q}$. If $d$ is the discriminant of $K$ and $d=d_{1} d_{2} \cdots d_{t}$ is the factorization of $d$ into the product of fundamental prime discriminants, then $K_{g}=\mathbf{Q}\left(\sqrt{d_{1}}, \sqrt{d_{2}}, \ldots, \sqrt{d_{t}}\right)$, and we have

$$
\mathbf{Q} \subset K \subseteq K_{g} \subseteq K_{1} \subseteq\left(K_{g}\right)_{1} \subseteq K_{2},
$$

which implies

$$
\left[K_{2}: \mathrm{Q}\right]=\left[K_{2}:\left(K_{g}\right)_{1}\right]\left[\left(K_{g}\right)_{1}: \mathrm{Q}\right]=\left[K_{2}:\left(K_{g}\right)_{1}\right] h\left(K_{g}\right)\left[K_{g}: \mathrm{Q}\right] .
$$

As $K_{g}$ is a multi-quadratic number field, $h\left(K_{g}\right)$ can be calculated by the method in [51], and we may expect that $\left[K_{2}:\left(K_{g}\right)_{1}\right]$ is small for fields we consider on the ground of the following proposition ( $\S 4$, Proposition 2 ).

Proposition. Let $L$ be the Hilbert class field of the genus field $K_{g}$ of an imaginary abelian number field $K$. Then for any prime number $p$ with $p \nmid[L: \mathrm{Q}]$, the p-class group $\mathrm{Cl}^{(p)}(L)$ of $L$ is trivial or noncyclic.

For all $K$ with $|d|<1000$ such that $h\left(K_{g}\right)>h(K) /\left[K_{g}: K\right]$, which is equivalent to $\left(K_{g}\right)_{1} \supsetneqq K_{1}$, we have $K_{2}=\left(K_{g}\right)_{1}$. For $h\left(K_{g}\right)>h(K) /\left[K_{g}\right.$ : $K], h(K)$ must necessarily be even. Now $h(K)$ is even if and only if $d$ has 
(at least) two distinct prime factors, however, for most $K$, this inequality holds. In fact, if a quadratic subfield $\neq K$ of $K_{g}$ has class number divisible by an odd prime $p$, then we have $h\left(K_{g}\right) \geqq p h(K) /\left[K_{g}: K\right]$. Thus, the following problem arises naturally:

Problem. Characterize the imaginary quadratic number fields $K$ with $K_{2}=\left(K_{g}\right)_{1} \neq K_{1}$.

The author expects that this problem can be settled group-theoretically and a similar problem can be posed for real quadratic number fields. We will discuss this problem in the Appendix 2.

For 12 fields $K$ with $|d|<1000$ for which we have verified $K_{u r} \supsetneqq\left(K_{g}\right)_{1}$, there exists an $S_{4}$-extension $M$ of $\mathrm{Q}$ such that $K_{g} M / K_{g}$ is an unramified $A_{4}$-extension, where $S_{4}$ (resp. $A_{4}$ ) denotes the symmetric (resp. alternating) group of degree four, and therefore $K_{u r} \supseteqq\left(K_{g}\right)_{1} M \supsetneqq\left(K_{g}\right)_{1}$. Except for $\mathrm{Q}(\sqrt{-856})$ and $\mathrm{Q}(\sqrt{-996})$, we can charaterize such $K$ simply as $d_{E} \mid d$ for some quartic number field $E$ : If the discriminant of $d$ of $K$ is divisible by the discriminant $d_{E}$ of a quartic number field $E$, then $K_{g}$ has an unramified $A_{4}$-extension. Then the normal closure of $E$ is an $S_{4}$-extension of $\mathbf{Q}$ unramified at all finite primes over its quadratic subfield $\mathrm{Q}\left(\sqrt{d_{E}}\right)$. Since $d_{E} \mid d, K \mathrm{Q}\left(\sqrt{d_{E}}\right) / K$ is unramified by genus theory and therefore $K_{g} M / K_{g}$ is an unramified $A_{4}$-extension. Also for the fields $\mathbf{Q}(\sqrt{-856})$ and $\mathrm{Q}(\sqrt{-996})$, we can find $S_{4}$-extensions of $\mathrm{Q}$ that give their unramified $S_{4}$-extensions by composition. (For details, see $\S 7$.) Therefore, data for quartic number fields are useful for our study. Thus, $K=\mathbf{Q}(\sqrt{d})$ with $|d|<1000$, can be classified simply as follows:

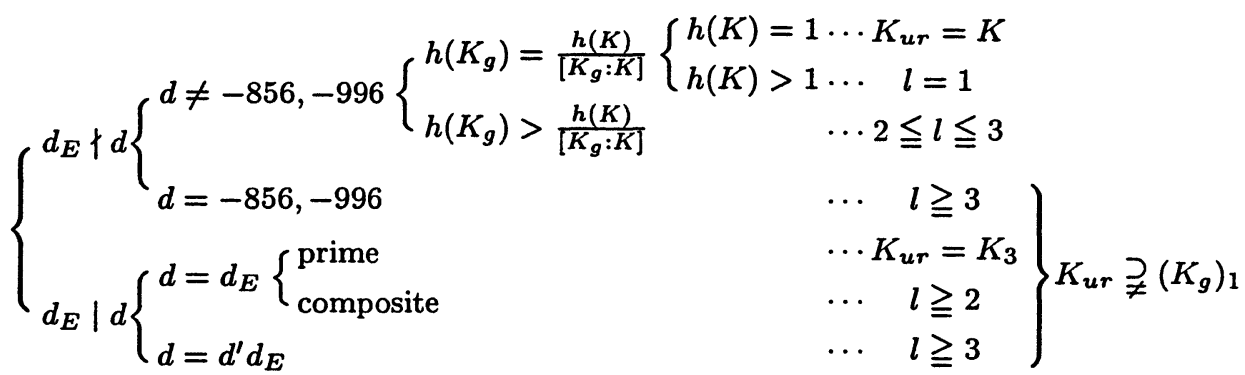

Here, by " $d_{E} \mid d$ " (resp. " $d_{E} \nmid d$ ") we mean " $d$ is divisible by $d_{E}$ for some quartic number field" (resp. " $d$ is never divisible by the discriminant $d_{E}$ for any quartic number field"), and in the factorization of $d=d^{\prime} d_{E}, d^{\prime}$ denotes a fundamental quadratic discriminant. We expect that $K_{u r}=K_{l}$ holds for all $K$ with $|d|<1507$, because we expect that $Q(\sqrt{-1507})$ is the first $K$ having an unramified nonsolvable Galois extension (see below). This actually holds for all $K$ with $|d|<1000$ and $l \geqq 2$. We also expect that 
for all fields $K$ with $d_{E} \nmid d \neq-856,-996, K_{u r}=\left(K_{g}\right)_{1}$ holds and that all inequalities for $l$ are equalties. If our expectation is true, the classification above can be replaced by the following:

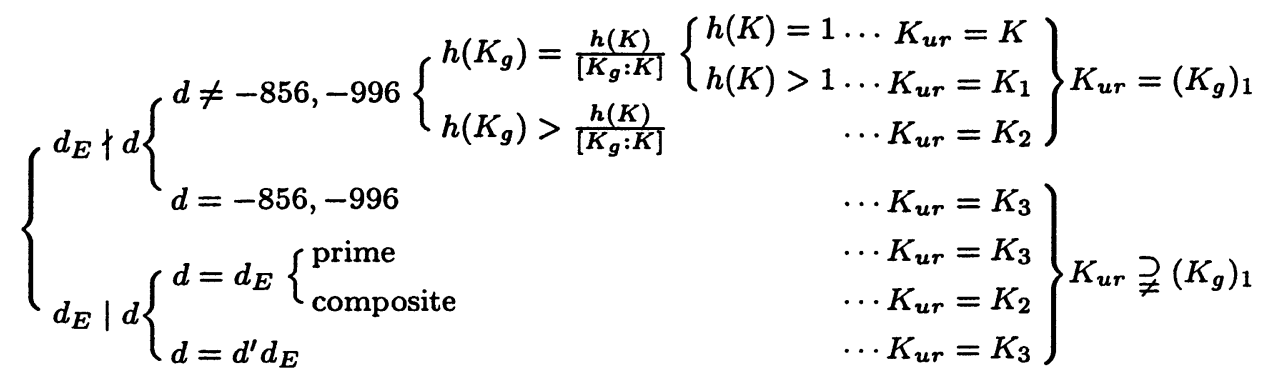

Though, there are possible exceptions, the author think that this classification is meaningful because this is complete for $|d| \leqq 719$ and even if an exception would occur for $|d|>719$, modification is easy.

For most $K$ we considered, $K_{u r}=K_{l}$ is verified. Thus, the following natural question arises: What is the first imaginary quadratic number field having an unramified nonsolvable Galois extension ? (What is the first $K$ with $K_{u r} \neq K_{l}$ ?) Recent data for quintic number fields [3,44] enable us to give a partial answer ( $\S 8$, Proposition 8 ):

Proposition. The field $\mathbf{Q}(\sqrt{-1507})$ is the first imaginary quadratic number field having an unramified $A_{5}$-extension which is normal over $\mathbf{Q}$ in the sense that none of $\mathbf{Q}(\sqrt{d})$ of discriminant $d$ with $0>d>-1507$ has such an extension.

We expect that the field $\mathbf{Q}(\sqrt{-1507})$ gives the answer to the question above.

For the determination of the structure of $\operatorname{Gal}\left(K_{u r} / K\right)$, the results on 2-class field towers due to $H$. Kisilevsky [25], F. Lemmermeyer [29, 31], and E. Benjamin, F. Lemmermeyer, and C. Snyder [5] are very helpful. They give us information on the structure of the Galois group $\operatorname{Gal}\left(K_{2}^{(2)} / K\right)$ of the second Hilbert 2-class field $K_{2}^{(2)}$ of $K$ over $K$ in many cases.

Now we explain the notations in our table. In the simple expressions of $K_{1}$ and $K_{2}, \alpha_{i}, \beta_{i}$ and $\gamma_{i}$ denote any algebraic numbers generating the $i$ th cubic number field of signature $(1,1)$, the $i$ th quartic number field of signature $(2,1)$ with Galois group isomorphic to $S_{4}$, and the $i$ th quintic number field of signature $(1,2)$ with Galois group isomorphic to $D_{5}$, respectively, where we consider that the number fields of each signature and each type (of Galois group of normal closure) are numbered up to conjugacy by absolute values of discriminants. (Here we do not need to consider nonisomorphic fields with same discriminants.) 
$G$ denotes the Galois group $\operatorname{Gal}\left(K_{u r} / K\right)$. As usual, $C_{n}$ is the cyclic group of order $n, V_{4}$ is the four group, that is, $V_{4}=C_{2}^{2}=C_{2} \times C_{2}, D_{n}$ $(n \geqq 3)$ is the dihedral group of order $2 n, Q_{4 n}(n \geqq 2)$ is the generalized quaternion group of order $4 n$, and $S D_{8 n}(n \geqq 2)$ is the semi-dihedral group of order $8 n$ :

$$
\begin{aligned}
D_{n} & =\left\langle a, b \mid a^{n}=b^{2}=1, b^{-1} a b=a^{-1}\right\rangle, \\
Q_{4 n} & =\left\langle a, b \mid a^{2 n}=1, b^{2}=a^{n}, b^{-1} a b=a^{-1}\right\rangle, \\
S D_{8 n} & =\left\langle a, b \mid a^{4 n}=b^{2}=1, b^{-1} a b=a^{2 n-1}\right\rangle .
\end{aligned}
$$

$I_{n}^{2 m}(m \geqq 2, n \geqq 3)$ denotes the group of order $2 m n$ given by

$$
\left\langle a, b \mid a^{2 m}=b^{n}=1, a^{-1} b a=b^{-1}\right\rangle .
$$

$M_{2^{n}}(n \geqq 4)$ denotes the modular group of order $2^{n}$ given by

$$
\left\langle a, b \mid a^{2^{n-1}}=b^{2}=1, b^{-1} a b=a^{2^{n-2}+1}\right\rangle .
$$

$\widetilde{A_{4}}$ is the double cover of $A_{4}: \widetilde{A_{4}} \cong \mathrm{SL}(2,3)$.

For some 2-groups we use designations given in the table by M. Hall and J. K. Senior [17]. We note that T. W. Sag and J. W. Wamsley give minimal presentations for all 2-groups of orders $\leqq 2^{6}$ [42]. For simplicity, for some of them we use the following designations used in [5]. $\Gamma_{m, t}^{1}$ denotes the group of order $2^{m+t+1}$ given by

$$
\left\langle a, b \mid a^{4}=b^{2^{m}}=1, c=[a, b], a^{2}=c^{2^{t-1}},[a, c]=c^{2},[a b, c]=1\right\rangle .
$$

$\Gamma_{m, t}^{2}$ denotes the group of order $2^{m+t+1}$ given by

$$
\left\langle a, b \mid a^{4}=1, c=[a, b], a^{2}=b^{2^{m}}=c^{2^{t-1}},[a, c]=c^{2},[a b, c]=1\right\rangle .
$$

We note that $\Gamma_{3,2}^{2}=64 \Gamma_{3} p, \Gamma_{2,3}^{1}=64 \Gamma_{8} c_{2}, \Gamma_{3,2}^{1}=64 \Gamma_{3} n_{2}, \Gamma_{2,3}^{2}=64 \Gamma_{8} e$.

The organization of this paper is as follows: In Section 2, we review how to obtain an upper bound for $\left[K_{u r}: K\right]$ by using discriminant bounds. In Section 3, we review some results on class number relations. In Section 4 , we describe the information on the structure of class groups that can be obtained by considering the action of Galois groups on class groups. In Section 5, we review some results on class field towers. In Section 6, we describe how to determine $\operatorname{Gal}\left(K_{u r} / K\right)$ for selected values of $d$. In Section 7, we describe fields having an unramified extension not contained 
in $\left(K_{g}\right)_{1}$. In Section 8, we describe unramified nonsolvable Galois extensions of imaginary quadratic number fields. In the Appendix 1, we explain how to calculate class numbers of $S_{4}$-extensions of $\mathbf{Q}$. In the Appendix 2, we discuss the problem of characterizing quadratic number fields $K$ with $K_{2}=\left(K_{g}\right)_{1} \neq K_{1}$.

Acknowledgements. The author thanks Prof. R. Schoof for useful advices [43]. He also thanks Dr. F. Lemmermeyer for information on his results [32].

Table of imaginary quadratic number fields $K=\mathbf{Q}(\sqrt{d}),|d| \leqq 719$ with $K_{u r} \neq K_{1}$

\begin{tabular}{|c|c|l|l|c|c|}
\hline$-d$ & $\mathrm{Cl}(K)$ & \multicolumn{1}{|c|}{$K_{1}$} & \multicolumn{1}{|c|}{$K_{2}$} & $l$ & $G$ \\
\hline 115 & $C_{2}$ & $K(\sqrt{5})$ & $K_{1}\left(\alpha_{1}\right)$ & $D_{3}$ \\
120 & $V_{4}$ & $K(\sqrt{-3}, \sqrt{5})$ & $K_{1}(\sqrt{(2 \sqrt{2}+\sqrt{5})(2+\sqrt{5})})$ & 2 & $Q_{8}$ \\
155 & $C_{4}$ & $K(\sqrt{(-1+5 \sqrt{5}) / 2})$ & $K_{1}\left(\alpha_{2}\right)$ & 2 & $Q_{12}$ \\
184 & $C_{4}$ & $K(\sqrt{-3}+4 \sqrt{2})$ & $K_{1}\left(\alpha_{1}\right)$ & 2 & $Q_{12}$ \\
195 & $V_{4}$ & $K(\sqrt{-3}, \sqrt{5})$ & & 2 & $Q_{16}$ \\
235 & $C_{2}$ & $K(\sqrt{5})$ & $K_{1}\left(\gamma_{1}\right)$ & 2 & $D_{5}$ \\
248 & $C_{8}$ & & 2 & $I_{3}^{8}$ \\
255 & $C_{6} \times C_{2}\left(\alpha_{2}\right)$ & $K(\sqrt{5}, \sqrt[3]{(9+\sqrt{85}) / 2})$ & $K_{1}(\sqrt{(5+2 \sqrt{-3})(2+\sqrt{5})})$ & 2 & $Q_{8} \times C_{3}$ \\
260 & $C_{4} \times C_{2}$ & $K(\sqrt{5}, \sqrt{8+\sqrt{65})}$ & & 2 & $M_{16}$ \\
276 & $C_{4} \times C_{2}$ & $K(\sqrt{-1}, \sqrt{13+8 \sqrt{3}})$ & $K_{1}\left(\alpha_{1}\right)$ & 2 & $Q_{12} \times C_{2}$ \\
280 & $V_{4}$ & $K(\sqrt{-7}, \sqrt{5})$ & & 2 & $Q_{16}$ \\
283 & $C_{3}$ & $K\left(\alpha_{31}\right)$ & $K_{1}\left(\beta_{1}\right)$ & 3 & $\mathcal{A}_{4}$ \\
295 & $C_{8}$ & & 2 & $I_{3}^{8}$ \\
299 & $C_{8}$ & & 2 & $I_{3}^{8}$ \\
312 & $V_{4}$ & $K(\sqrt{-3}, \sqrt{2})$ & 2 & $Q_{16}$ \\
331 & $C_{3}$ & $K\left(\alpha_{36}\right)$ & 3 & $\widetilde{A_{4}}$ \\
340 & $V_{4}$ & $K(\sqrt{-1}, \sqrt{5})$ & $K_{1}\left(\alpha_{1}\right)$ & 2 & $S D_{16}$ \\
355 & $C_{4}$ & $K(\sqrt{-3}+4 \sqrt{5})$ & & 2 & $Q_{28}$ \\
372 & $V_{4}$ & $K(\sqrt{-1}, \sqrt{-3})$ & $K_{1}\left(\alpha_{2}\right)$ & 2 & $D_{6}$ \\
376 & $C_{8}$ & & $K_{1}\left(\gamma_{1}\right)$ & 2 & $I_{5}^{8}$ \\
391 & $C_{14}$ & & $K_{1}\left(\alpha_{1}\right)$ & 2 & $D_{3} \times C_{7}$ \\
395 & $C_{8}$ & & $K_{1}\left(\gamma_{2}\right)$ & $I_{5}^{8}$ \\
403 & $C_{2}$ & $K(\sqrt{13})$ & $K_{1}\left(\alpha_{2}\right)$ & 2 & $D_{3}$ \\
408 & $V_{4}$ & $K(\sqrt{-3}, \sqrt{2})$ & $K_{1}\left(\alpha_{6}\right)$ & $D_{4}$ \\
415 & $C_{10}$ & $K\left(\sqrt{5}, \gamma_{18}\right)$ & 2 & $D_{3} \times C_{5}$ \\
420 & $C_{2}^{3}$ & $K(\sqrt{-1}, \sqrt{-3}, \sqrt{5})$ & & $32 \Gamma_{4} c_{3}$ \\
\hline
\end{tabular}


Continued (under GRH)

\begin{tabular}{|c|c|c|c|c|c|}
\hline$-d$ & $\mathrm{Cl}(K)$ & $K_{1}$ & $K_{2}$ & $l$ & $G$ \\
\hline 435 & $V_{4}$ & $K(\sqrt{-3}, \sqrt{5})$ & & 2 & $Q_{16} \times C_{3}$ \\
\hline 440 & $C_{6} \times C_{2}$ & $\left(\sqrt{2}, \sqrt{5}, \alpha_{50}\right)$ & & 2 & $Q_{16} \times C_{3}$ \\
\hline 455 & $C_{10} \times C_{2}$ & $K\left(\sqrt{-7}, \sqrt{5}, \gamma_{21}\right)$ & & 2 & $Q_{8} \times C_{5}$ \\
\hline 472 & $C_{6}$ & $K\left(\sqrt{2}, \alpha_{4}\right)$ & $K_{1}\left(\alpha_{4}\right)$ & 2 & $D_{3} \times C_{3}$ \\
\hline 483 & $V_{4}$ & $K(\sqrt{-3}, \sqrt{-7})$ & $K_{1}\left(\alpha_{1}\right)$ & 2 & $D_{6}$ \\
\hline 491 & $C_{9}$ & & $K_{1}\left(\beta_{3}\right)$ & 3 & $Q_{8} \times C_{9}$ \\
\hline 515 & $C_{6}$ & $K\left(\sqrt{5}, \alpha_{60}\right)$ & $K_{1}\left(\gamma_{3}\right)$ & 2 & $D_{5} \times C_{3}$ \\
\hline 520 & $V_{4}$ & $K(\sqrt{-2}, \sqrt{5})$ & & 2 & $Q_{24}$ \\
\hline 527 & $C_{18}$ & & $K_{1}\left(\alpha_{2}\right)$ & 2 & $D_{3} \times C_{9}$ \\
\hline 535 & $C_{14}$ & & $K_{1}\left(\alpha_{9}\right)$ & 2 & $D_{3} \times C_{7}$ \\
\hline 552 & $C_{4} \times C_{2}$ & $K(\sqrt{-3}, \sqrt{-1+2 \sqrt{6}})$ & $K_{1}\left(\alpha_{1}\right)$ & 2 & $Q_{12} \times C_{2}$ \\
\hline 555 & $V_{4}$ & $K(\sqrt{-3}, \sqrt{5})$ & & 2 & $Q_{32}$ \\
\hline 563 & $C_{9}$ & & $K_{1}\left(\beta_{4}\right)$ & 3 & $Q_{8} \rtimes C_{9}$ \\
\hline 564 & $C_{4} \times C_{2}$ & $K(\sqrt{-1}, \sqrt{1+4 \sqrt{3}})$ & $K_{1}\left(\gamma_{1}\right)$ & 2 & $Q_{20} \times C_{2}$ \\
\hline 568 & $C_{4}$ & $K(\sqrt{-1+6 \sqrt{2}})$ & & 2 & $Q_{28}$ \\
\hline 580 & $C_{4} \times C_{2}$ & $K(\sqrt{5}, \sqrt{12+\sqrt{145}})$ & & 2 & $32 \Gamma_{3} f \ltimes C_{3}$ \\
\hline 595 & $V_{4}$ & $K(\sqrt{-7}, \sqrt{5})$ & & 2 & $Q_{40}$ \\
\hline 611 & $C_{10}$ & $K\left(\sqrt{13}, \gamma_{28}\right)$ & $K_{1}\left(\gamma_{1}\right)$ & 2 & $D_{5} \times C_{5}$ \\
\hline 632 & $C_{8}$ & & $K_{1}\left(\gamma_{2}\right)$ & 2 & $I_{5}^{8}$ \\
\hline 635 & $C_{10}$ & $K\left(\sqrt{5}, \gamma_{31}\right)$ & $K_{1}\left(\gamma_{5}\right)$ & 2 & $D_{5} \times C_{5}$ \\
\hline 643 & $C_{3}$ & $K\left(\alpha_{72}\right)$ & $K_{1}\left(\beta_{5}\right)$ & 3 & $\widetilde{A_{4}}$ \\
\hline 644 & $C_{8} \times C_{2}$ & & $K_{1}\left(\alpha_{1}\right)$ & 2 & $D_{3} \times C_{8}$ \\
\hline 651 & $C_{4} \times C_{2}$ & $K(\sqrt{-7}, \sqrt{(13+\sqrt{217}) / 2})$ & $K_{1}\left(\alpha_{2}\right)$ & 2 & $D_{3} \times C_{4}$ \\
\hline 655 & $C_{12}$ & $K\left(\sqrt{7+6 \sqrt{5}}, \alpha_{75}\right)$ & $K_{1}\left(\gamma_{6}\right)$ & 2 & $Q_{20} \times C_{3}$ \\
\hline 660 & $C_{2}^{3}$ & $K(\sqrt{-1}, \sqrt{-3}, \sqrt{5})$ & & 2 & $64 \Gamma_{15} f_{2}$ \\
\hline 663 & $C_{8} \times C_{2}$ & & & 2 & $\Gamma_{3,2}^{2}$ \\
\hline 664 & $C_{10}$ & $K\left(\sqrt{2}, \gamma_{32}\right)$ & $K_{1}\left(\alpha_{6}\right)$ & 2 & $D_{3} \times C_{5}$ \\
\hline 667 & $C_{4}$ & $K(\sqrt{(-13+3 \sqrt{29}) / 2})$ & $K_{1}\left(\alpha_{1}\right)$ & 2 & $Q_{12}$ \\
\hline 680 & $C_{6} \times C_{2}$ & $K\left(\sqrt{-2}, \sqrt{5}, \alpha_{80}\right)$ & & 2 & $Q_{16} \times C_{3}$ \\
\hline 687 & $C_{12}$ & $K\left(\sqrt{(11+\sqrt{229}) / 2}, \alpha_{81}\right)$ & & 3 & $\left(A_{4} \rtimes C_{4}\right) \times C_{3}$ \\
\hline 695 & $C_{24}$ & & $K_{1}\left(\alpha_{13}\right)$ & 2 & $I_{3}^{8} \times C_{3}$ \\
\hline 696 & $C_{6} \times C_{2}$ & $K(\sqrt{2}, \sqrt[3]{99+13 \sqrt{58}})$ & & 2 & $Q_{24} \times C_{3}$ \\
\hline 708 & $V_{4}$ & $K(\sqrt{-1}, \sqrt{-3})$ & $K_{1}\left(\alpha_{4}\right)$ & 2 & $D_{6}$ \\
\hline 715 & $V_{4}$ & $K(\sqrt{-11}, \sqrt{5})$ & & 2 & $Q_{16} \times C_{5}$ \\
\hline
\end{tabular}


Table of $\operatorname{Gal}\left(K_{u r} / K\right)$ for $723 \leqq|d|<1000$ (not complete)

\begin{tabular}{|c|c|c|c|c|c|}
\hline$-d$ & $\mathrm{Cl}(K)$ & $\overline{K_{1}}$ & $\overline{K_{2}}$ & $l$ & $G$ \\
\hline 723 & $C_{4}$ & $K(\sqrt{(-7+3 \sqrt{241}) / 2})$ & & 1 & $?$ \\
\hline 724 & $C_{10}$ & $K\left(\sqrt{-1}, \gamma_{37}\right)$ & & 1 & $C_{10}$ \\
\hline 727 & $C_{13}$ & & & 1 & $C_{13}$ \\
\hline 728 & $C_{6} \times C_{2}$ & $K\left(\sqrt{-7}, \sqrt{2}, \alpha_{86}\right)$ & & 2 & $Q_{16} \times C_{3}$ \\
\hline 731 & $C_{12}$ & $K\left(\sqrt{-5+2 \sqrt{17}}, \alpha_{87}\right)$ & & $\geqq 2$ & $?$ \\
\hline 739 & $C_{5}$ & $K\left(\gamma_{38}\right)$ & & 1 & $C_{5}$ \\
\hline 740 & $C_{8} \times C_{2}$ & & & 2 & $M_{32}$ \\
\hline 743 & $C_{21}$ & & & 1 & $C_{21}$ \\
\hline 744 & $C_{6} \times C_{2}$ & $K(\sqrt{-2}, \sqrt[3]{63+8 \sqrt{62}})$ & $K_{1}\left(\alpha_{2}\right)$ & 2 & $D_{3} \times C_{6}$ \\
\hline 751 & $C_{15}$ & $K\left(\alpha_{91}, \gamma_{39}\right)$ & $K_{1}\left(\beta_{8}\right)$ & 3 & $\widetilde{A_{4}} \times C_{5}$ \\
\hline 755 & $C_{12}$ & $K\left(\sqrt{13+8 \sqrt{5}}, \alpha_{92}\right)$ & & 2 & $Q_{28} \times C_{3}$ \\
\hline 759 & $C_{12} \times C_{2}$ & $K\left(\sqrt{-3}, \sqrt{(-5+\sqrt{69}) / 2}, \alpha_{94}\right)$ & $K_{1}\left(\alpha_{1}\right)$ & 2 & $D_{3} \times C_{12}$ \\
\hline 760 & $V_{4}$ & $K(\sqrt{2}, \sqrt{5})$ & & 2 & $Q_{32} \ltimes C_{3}$ \\
\hline 763 & $C_{4}$ & $K(\sqrt{(-9+\sqrt{109}) / 2})$ & & 1 & $?$ \\
\hline 767 & $C_{22}$ & & $K_{1}\left(\alpha_{4}\right)$ & 2 & $D_{3} \times C_{11}$ \\
\hline 771 & $C_{6}$ & $K(\sqrt[3]{16+\sqrt{257}})$ & & $\geqq 3$ & ? \\
\hline 772 & $C_{4}$ & $K(\sqrt{(-7+\sqrt{193}) / 2})$ & & 1 & $?$ \\
\hline 776 & $C_{20}$ & $K\left(\sqrt{(-5+\sqrt{97}) / 2}, \gamma_{40}\right)$ & & 1 & $C_{20}$ \\
\hline 779 & $C_{10}$ & $K\left(\sqrt{-19}, \gamma_{41}\right)$ & & 1 & $C_{10}$ \\
\hline 787 & $C_{5}$ & $K\left(\gamma_{42}\right)$ & & 1 & $?$ \\
\hline 788 & $C_{10}$ & $K\left(\sqrt{-1}, \gamma_{43}\right)$ & & 1 & $C_{10}$ \\
\hline 791 & $C_{32}$ & & & 1 & $C_{32}$ \\
\hline 795 & $V_{4}$ & $K(\sqrt{-3}, \sqrt{5})$ & & 2 & $Q_{40}$ \\
\hline 799 & $C_{16}$ & & $K_{1}\left(\gamma_{1}\right)$ & 2 & $I_{5}^{16}$ \\
\hline 803 & $C_{10}$ & $K\left(\sqrt{-11}, \gamma_{44}\right)$ & & 1 & $C_{10}$ \\
\hline 804 & $C_{6} \times C_{2}$ & $K(\sqrt{-1}, \sqrt[3]{22+2 \sqrt{67}})$ & & 1 & $C_{6} \times C_{2}$ \\
\hline 807 & $C_{14}$ & & & 1 & $C_{14}$ \\
\hline 808 & $C_{6}$ & $K\left(\sqrt{-2}, \alpha_{98}\right)$ & & 1 & $?$ \\
\hline 811 & $C_{7}$ & & & 1 & $C_{7}$ \\
\hline 815 & $C_{30}$ & $K\left(\sqrt{5}, \alpha_{100}, \gamma_{45}\right)$ & & 1 & $C_{30}$ \\
\hline 820 & $C_{4} \times C_{2}$ & $K(\sqrt{-1}, \sqrt{6+\sqrt{41}})$ & & 2 & $\Gamma_{2,3}^{1}$ \\
\hline 823 & $C_{9}$ & & & 1 & $C_{9}$ \\
\hline 824 & $C_{20}$ & $K\left(\sqrt{5+8 \sqrt{2}}, \gamma_{46}\right)$ & $K_{1}\left(\gamma_{3}\right)$ & 2 & $Q_{20} \times C_{5}$ \\
\hline 827 & $C_{7}$ & & & 1 & $C_{7}$ \\
\hline 831 & $C_{28}$ & & & 1 & $C_{28}$ \\
\hline 835 & $C_{6}$ & $K\left(\sqrt{5}, \alpha_{102}\right)$ & & 2 & $D_{11} \times C_{3}$ \\
\hline 836 & $C_{10} \times C_{2}$ & $K\left(\sqrt{-1}, \sqrt{-11}, \gamma_{47}\right)$ & & 1 & $C_{10} \times C_{2}$ \\
\hline 839 & $C_{33}$ & & & 1 & $C_{33}$ \\
\hline 840 & $C_{2}^{3}$ & $K(\sqrt{-2}, \sqrt{-3}, \sqrt{5})$ & & 2 & $32 \Gamma_{4} d$ \\
\hline 843 & $C_{6}$ & $K(\sqrt[3]{(5+\sqrt{281}) / 2})$ & & 1 & $?$ \\
\hline
\end{tabular}


Continued

\begin{tabular}{|c|c|c|c|c|c|}
\hline$-d$ & $\mathrm{Cl}(K)$ & $K_{1}$ & $K_{2}$ & $l$ & $G$ \\
\hline 851 & $C_{10}$ & $K\left(\sqrt{-23}, \gamma_{49}\right)$ & $K_{1}\left(\alpha_{4}\right)$ & 2 & $D_{3} \times C_{5}$ \\
\hline 852 & $C_{4} \times C_{2}$ & $K(\sqrt{-1}, \sqrt{2+5 \sqrt{3}})$ & & 2 & $Q_{28} \times C_{2}$ \\
\hline 856 & $C_{6}$ & $K\left(\sqrt{2}, \alpha_{105}\right)$ & $K_{1}\left(\alpha_{9}\right)$ & $\geqq 3$ & $?$ \\
\hline 859 & $C_{7}$ & & & 1 & $C_{7}$ \\
\hline 863 & $C_{21}$ & & & 1 & $C_{21}$ \\
\hline 868 & $C_{4} \times C_{2}$ & $K(\sqrt{-1}, \sqrt{9+4 \sqrt{7}})$ & $K_{1}\left(\alpha_{2}\right)$ & 2 & $Q_{12} \times C_{2}$ \\
\hline 871 & $C_{22}$ & & & 1 & $C_{22}$ \\
\hline 872 & $C_{10}$ & $K\left(\sqrt{-2}, \gamma_{50}\right)$ & & 1 & $C_{10}$ \\
\hline 879 & $C_{22}$ & & & 1 & $C_{22}$ \\
\hline 883 & $C_{3}$ & $K\left(\alpha_{109}\right)$ & & 1 & $?$ \\
\hline 884 & $C_{8} \times C_{2}$ & & & 2 & $\Gamma_{3,2}^{1}$ \\
\hline 887 & $C_{29}$ & & & 1 & $C_{29}$ \\
\hline 888 & $C_{6} \times C_{2}$ & $K(\sqrt{2}, \sqrt[3]{43+5 \sqrt{74}})$ & & 2 & $Q_{32} \times C_{3}$ \\
\hline 895 & $C_{16}$ & & $K_{1}\left(\gamma_{9}\right)$ & 2 & $I_{5}^{16}$ \\
\hline 899 & $C_{14}$ & & $K_{1}\left(\alpha_{2}\right)$ & 2 & $D_{3} \times C_{7}$ \\
\hline 903 & $C_{8} \times C_{2}$ & & & 1 & $C_{8} \times C_{2}$ \\
\hline 904 & $C_{8}$ & & & 1 & $?$ \\
\hline 907 & $C_{3}$ & $K\left(\alpha_{112}\right)$ & & 1 & $?$ \\
\hline 911 & $C_{31}$ & & & 1 & $C_{31}$ \\
\hline 915 & $C_{4} \times C_{2}$ & $K(\sqrt{-3}, \sqrt{(-1+\sqrt{61}) / 2})$ & & 2 & $\Gamma_{2,3}^{2}$ \\
\hline 916 & $C_{10}$ & $K\left(\sqrt{-1}, \gamma_{52}\right)$ & & $\geqq 3$ & ? \\
\hline 919 & $C_{19}$ & & & 1 & $C_{19}$ \\
\hline 920 & $C_{10} \times C_{2}$ & $K\left(\sqrt{2}, \sqrt{5}, \gamma_{53}\right)$ & & 2 & $\left(Q_{16} \ltimes C_{3}\right) \times C_{5}$ \\
\hline 923 & $C_{10}$ & $K\left(\sqrt{13}, \gamma_{54}\right)$ & & 2 & $D_{7} \times C_{5}$ \\
\hline 932 & $C_{12}$ & $K\left(\sqrt{(13+\sqrt{233}) / 2}, \alpha_{115}\right)$ & & 1 & $?$ \\
\hline 935 & $C_{14} \times C_{2}$ & & & 2 & $Q_{16} \times C_{7}$ \\
\hline 939 & $C_{8}$ & & & 1 & $?$ \\
\hline 943 & $C_{16}$ & & $K_{1}\left(\alpha_{4}\right)$ & 2 & $I_{3}^{16}$ \\
\hline 947 & $C_{5}$ & $K\left(\gamma_{55}\right)$ & & 1 & $?$ \\
\hline $\begin{array}{l}948 \\
951\end{array}$ & $\begin{array}{c}C_{6} \times C_{2} \\
C_{26}\end{array}$ & $K(\sqrt{-1}, \sqrt[3]{80+9 \sqrt{79}})$ & $K_{1}\left(\gamma_{2}\right)$ & $\begin{array}{l}2 \\
1\end{array}$ & $\begin{array}{c}D_{5} \times C_{6} \\
C_{26}\end{array}$ \\
\hline 952 & $C_{4} \times C_{2}$ & $K(\sqrt{-7}, \sqrt{9+10 \sqrt{2}})$ & & $\geqq 2$ & $?$ \\
\hline 955 & $C_{4}$ & $K(\sqrt{(-9+13 \sqrt{5}) / 2})$ & & 2 & $Q_{52}$ \\
\hline 959 & $C_{36}$ & & & 1 & $C_{36}$ \\
\hline 964 & $C_{12}$ & $K\left(\sqrt{(-15+\sqrt{241}) / 2}, \alpha_{119}\right)$ & & 1 & $?$ \\
\hline 967 & $C_{11}$ & & & 1 & $C_{11}$ \\
\hline 971 & $C_{15}$ & $K\left(\alpha_{120}, \gamma_{57}\right)$ & & 1 & $?$ \\
\hline 979 & $C_{8}$ & & & 1 & $?$ \\
\hline
\end{tabular}


Continued

\begin{tabular}{|c|c|c|r|r|c|}
\hline$-d$ & $\mathrm{Cl}(K)$ & $K_{1}$ & $K_{2}$ & $l$ & $G$ \\
\hline 983 & $C_{27}$ & & & 1 & $C_{27}$ \\
984 & $C_{6} \times C_{2}$ & $K(\sqrt{2}, \sqrt[3]{9+\sqrt{82}})$ & & $\geqq 2$ & $?$ \\
987 & $C_{4} \times C_{2}$ & $K(\sqrt{-3}, \sqrt{(-1+3 \sqrt{21}}) / 2)$ & & $\geqq 2$ & $?$ \\
991 & $C_{17}$ & & & 1 & $C_{17}$ \\
995 & $C_{8}$ & & & $I_{9}^{8}$ \\
996 & $C_{6} \times C_{2}$ & $K(\sqrt{-1}, \sqrt[3]{82+9 \sqrt{83}})$ & $K_{1}\left(\alpha_{2}\right)$ & $\geqq 3$ & $?$ \\
\hline
\end{tabular}

Supplements. We expect that all inequalities for $l$ in our table are equalities. For this expectation we give the following supplemental data.

For $K=\mathbf{Q}(\sqrt{-731}) \cdot \operatorname{Gal}\left(K_{2} / K\right) \cong A_{4} \times C_{4}$. If $K_{u r} \neq K_{2}, \operatorname{Gal}\left(K_{u r} / K_{2}\right)$ $\cong C_{2}$, or $C_{4}$.

For $K=\mathbf{Q}(\sqrt{-771}) . \operatorname{Gal}\left(K_{3} / K\right) \cong S_{4} \times C_{3}$. If $K_{u r} \neq K_{3}, \operatorname{Gal}\left(K_{u r} / K_{3}\right)$ $\cong C_{2}$, or $C_{4}$.

For $K=\mathrm{Q}(\sqrt{-856}) . \operatorname{Gal}\left(K_{2} / K\right) \cong D_{3} \times C_{3} . K_{2}$ has an unramified $V_{4}$-extension $L$ which is an $S_{4} \times C_{3}$-extension of $K$. If $K_{u r} \neq L$, then $\left[K_{u r}: L\right]=2$, or 4 .

For $K=\mathrm{Q}(\sqrt{-916}) \cdot \operatorname{Gal}\left(K_{2} / K\right) \cong D_{3} \times C_{5}$. Put $L=K \mathbf{Q}(\sqrt{229})_{w-u r}$, where $\mathbf{Q}(\sqrt{229})_{w-u r}$ denotes the maximal extension of $\mathbf{Q}(\sqrt{229})$ unramified at all finite primes, which is an $S_{4}$-extension of Q. (Cf. For $K=$ $\mathbf{Q}(\sqrt{-687}), K_{u r}=K_{1} \mathbf{Q}(\sqrt{229})_{w-u r}$. See $\S 7$. The index " $w$-ur" means "weakly-unramified".) $L$ is an unramified $V_{4}$-extension of $K_{2}$ which is an $S_{4} \times C_{5}$-extension of $K$. If $K_{u r} \neq L$, then $\left[K_{u r}: L\right]=2$, or 4 .

For $K=\mathrm{Q}(\sqrt{-952}) . \quad \mathrm{Gal}\left(K_{2} / K\right) \cong \Gamma_{2,2}^{2} \rtimes C_{5} . \quad$ (Note that $\Gamma_{2,2}^{2} \cong$ $C_{4} / C_{2}\left(=32 \Gamma_{3} e\right.$.) If $K_{u r} \neq K_{2}, \operatorname{Gal}\left(K_{u r} / K_{2}\right) \cong C_{2}$.

For $K=\mathbf{Q}(\sqrt{-984}) . \operatorname{Gal}\left(K_{2} / K\right) \cong Q_{8} \times C_{3}$. If $K_{u r} \neq K_{2}, \operatorname{Gal}\left(K_{u r} / K_{2}\right)$ $\cong C_{3}^{2}$, or $C_{5}^{2}$.

For $K=\mathbf{Q}(\sqrt{-987}) \cdot \operatorname{Gal}\left(K_{2} / K\right) \cong Q_{20} \times C_{2}$. If $K_{u r} \neq K_{2}, \operatorname{Gal}\left(K_{u r} / K_{2}\right)$ $\cong C_{2}^{4}$.

For $K=\mathrm{Q}(\sqrt{-996}) . \mathrm{Gal}\left(K_{2} / K\right) \cong D_{3} \times C_{6} . K_{2}$ has an unramified $V_{4^{-}}$ extension $L$ which is an $S_{4} \times C_{6}$-extension of $K$. We have $\left[K_{u r}: L\right] \leqq 32$ and the odd part of $\mathrm{Cl}(L)$ is trivial or isomorphic to $C_{3}^{2}, C_{3}^{3}$, or $C_{5}^{2}$. 


\section{UPPER BOUND FOR $\left[K_{u r}: K\right]$}

We review here how to obtain an upper bound for $\left[K_{u r}: K\right]$ for a given number field $K$ by using discriminant bounds.

In this section $K$ denotes an algebraic number field of finite degree. If we denote by $n_{K}$ the degree of $K$, the $n_{K}$ th root of the absolute value of the discriminant $d_{K}$ of $K$ is called root discriminant of $K$ and denoted by $r d_{K}$ :

$$
r d_{K}=\left|d_{K}\right|^{1 / n_{K}} .
$$

The following lemma is fundamental for our study.

LEMMA 1. Let $L / K$ be a finite extension of algebraic number fields. Then $r d_{L}=r d_{K}$ if and only if $L / K$ is unramified at all finite primes.

This can be easily proved by the transitive law of discriminants.

The following estimates for $r d=r d_{L}$ are known (see [39]): Unconditionally we have

$$
\begin{aligned}
r d & \geqq\left(4 \pi e^{1+C}\right)^{r_{1} / n}\left(4 \pi e^{C}\right)^{2 r_{2} / n}-O\left(n^{-3 / 2}\right) \\
& =(60.8395 \ldots)^{r_{1} / n}(22.3816 \ldots)^{2 r_{2} / n}-O\left(n^{-3 / 2}\right),
\end{aligned}
$$

as $n \rightarrow \infty$, where $C=0.5772 \ldots$ denotes Euler's constant, and $n=n_{L}$ and $r_{1}$ (resp. $r_{2}$ ) denotes the number of real (resp. imaginary) primes of $L$. From this, for all imaginary quadratic number fields $K=\mathbf{Q}(\sqrt{d}), d<0$ with $|d| \leqq 499,\left[K_{u r}: K\right]<\infty$. Under GRH we have

$$
\begin{aligned}
r d & \geqq\left(8 \pi e^{C+\pi / 2}\right)^{r_{1} / n}\left(8 \pi e^{C}\right)^{2 r_{2} / n}-O\left((\log n)^{-2}\right) \\
& =(215.3325 \ldots)^{r_{1} / n}(44.7632 \ldots)^{2 r_{2} / n}-O\left((\log n)^{-2}\right),
\end{aligned}
$$

as $n \rightarrow \infty$. From this, for all $K=\mathrm{Q}(\sqrt{d}), d<0$ with $|d| \leqq 2003$, $\left[K_{u r}: K\right]<\infty$.

By Lemma 1 we immediately obtain the following proposition, which tells us how to get an upper bound for $\left[K_{u r}: K\right]$.

Proposition 1. Let $B\left(n, r_{1}, r_{2}\right)$ ( $\mathbf{N} \ni n=r_{1}+2 r_{2}, r_{1}, r_{2}$ nonnegative integers) be the lower bound for the root discriminants of algebraic number fields $F$ of finite degrees $(\geqq n)$ such that $r_{i}(F) / n_{L}=r_{i} / n$ for $i=1,2$, where $r_{1}(F)$ (resp. $r_{2}(F)$ ) is the number of real (resp. imaginary) primes of $F$. Suppose that $K$ has an unramified normal extension $L$ of degree $m$. 
Let $H$ be a positive integer.

(i) If $r d_{K}<B\left(H m n_{K}, H m r_{1}(K), H m r_{2}(K)\right)$, then $\left[K_{u r}: L\right]<H$ and therefore $h(L)<H$. In particular, if $r d_{K}<B\left(2 m n_{K}, 2 m r_{1}(K), 2 m r_{2}(K)\right)$, then $K_{u r}=L$.

(ii) If $h(L)=1$ and $r d_{K}<B\left(60 m n_{K}, 60 m r_{1}(K), 60 m r_{2}(K)\right)$, then $K_{u r}=L$.

We note that the number 60 in (ii) is the minimal order of the finite nonsolvable groups, that is the order of $A_{5}$, the alternating group of degree five.

Thus, the knowledge of good lower bounds for $B\left(n, r_{1}, r_{2}\right)$ is very important for our study. The best known unconditional lower bounds for $B\left(n, r_{1}, r_{2}\right)$ can be found in the tables due to F. Diaz y Diaz [12]. If we assume GRH, much better lower bounds can be obtained. The best known conditional (GRH) lower bounds are found in the unpublished tables due to A. M. Odlyzko [38], which are copied in Martinet's expository paper [34].

All the imaginary quadratic number fields $K=\mathbf{Q}(\sqrt{d}),-d=3,4,7,8,11$, $19,43,67,163$ satisfy the condition in (ii) for $L=K$. In fact by the table in [12] we have $r d_{K} \leqq \sqrt{163}<B(60 \cdot 2,0,60 \cdot 1)$. Therefore none of these fields has any nontrivial unramified extension.

Let $K$ be an imaginary quadratic number field of small conductor. What unramified normal extension can we take as $L$ when we apply Proposition 1 ? Since known lower bounds for $B\left(n, r_{1}, r_{2}\right)$ grow large as $n$ grows large if $r_{2} / n$ is fixed, we need to take an extension $L$ of large degree. By the reason described in the Introduction it seems that the best choice is $L=\left(K_{g}\right)_{1}$, the Hilbert class field of the genus field $K_{g}$ of $K$ except when we can easily get an unramified extension of larger degree. In most cases we cannot conclude $K_{u r}=L$ only by (i) of Proposition 1, that is, we get only $\left[K_{u r}: L\right]<H$ for some $H \geqq 3$. Therefore we need to show $h(L)=1$ by class number calculation or using criteria for class number divisibility.

\section{Class number Relations}

In this section we review some results on class number relations on normal extensions of algebraic number fields. We will use them to calculate class numbers of fields of large degrees from class numbers of subfields.

Let $L / K$ be a (finite) normal extension of algebraic number fields and $G$ its Galois group. For any subgroup $H$ of $G$, we denote by $1_{H}^{G}$ the induced character of $G$ from the principal character of $H$. S. Kuroda [27] and R. Brauer [6] proved independently the following relation among the class 
numbers $h(M)$, the regulators $R(M)$, and the numbers $w_{2}(M)$ of roots of unity of 2-power orders of intermediate fields $M$ of $L / K$ : If we have a linear relation among $1_{H}^{G}$

$$
\sum_{H \leq G} a_{H} 1_{H}^{G}=0
$$

then we have the following relation

$$
\prod_{H \leq G}\left(\frac{h\left(L^{H}\right) R\left(L^{H}\right)}{w_{2}\left(L^{H}\right)}\right)^{a_{H}}=1 .
$$

Here, $L^{H}$ denotes the intermediate field of $L / K$ corresponding to $H$ by Galois theory.

We want to get $h(L)$ from the knowledge of $h(K)$ and $h(M)$ for intermediate fields $M$. When an arbitrary finite group $G$ is given, there does not exist necessarily such a nontrivial (that is, $a_{H} \neq 0$ for some $H$ ) linear relation. Even if there exists a nontrivial linear relation, it is difficult to use $(\dagger)$ in this form because it contains regulators. However, in some cases the product $\prod_{H} R\left(L^{H}\right)^{a_{H}}$ can be simplified into the form $\left[E_{L}: E\right] / q$ by elementary calculations, where $E_{L}$ is the group of units in $L, E$ is its subgroup generated by $E_{M}$ for some subfields $M$ of $L$, and $q$ is some power of a prime, and moreover $\left[E_{L}: E\right]$ is also some power of the prime dividing $q$. Such a simplified relation is often very useful for calculation of class numbers of fields of high degrees.

Let $G=\left\langle a, b \mid a^{2 n}=b^{2}=1, b^{-1} a b=a^{-1}\right\rangle$ be the dihedral group of order $2 n(n \geqq 2)$ (the four group if $n=2)$. Then we have the following relation:

$$
1_{\langle 1\rangle}^{G}+2 \cdot 1_{G}^{G}=1_{\langle b\rangle}^{G}+1_{\langle a\rangle}^{G}+1_{\langle a b\rangle}^{G} .
$$

From this and some elementary calculations, we get the following lemmas.

LEMMA 2. ([27, 28]) Let $L / K$ be a $V_{4}$-extension of algebraic number fields and $F, M$ and $N$ its three intermediate fields. Then we have the following class number relation:

$$
h(L)=\frac{\left[E_{L}: E_{F} E_{M} E_{N}\right]}{2^{2+r+v-t}} \cdot \frac{h(F) h(M) h(N)}{h(K)^{2}},
$$

where $r$ is the $\mathbf{Z}$-rank of $E_{K}, t$ is the number of infinite primes ramified in $L / K$, and $v \in\{0,1\}$ (for the definition of $v$, see [28]). Moreover, the index $\left[E_{L}: E_{F} E_{M} E_{N}\right]$ is a nonnegative power of 2 . 
The special case $K=\mathbf{Q}$ and $L \supset \mathbf{Q}(\sqrt{-1})$ of this is a classical result due to Dirichlet, which we can consider as a prototype. By this lemma we can calculate class numbers of some fields of degree 24 .

LEMMA 3. ([18, 19, 36]) Let $K$ be an imaginary quadratic number field or the rational number field $\mathrm{Q}$, and $p$ an odd prime number. Let $L$ be a $D_{p}$-extension of $K$. Let $M$ and $M^{\prime}$ be any two intermediate fields of $L / K$ with $[M: K]=\left[M^{\prime}: K\right]=p$ and $N$ the unique quadratic subextension of $L / K$. Then we have the following class number relation:

$$
h(L)=\frac{\left[E_{L}: E_{M} E_{M^{\prime}} E_{N}\right]}{p^{b}} \cdot \frac{h(M)^{2} h(N)}{h(K)^{2}},
$$

where $b=1$ if $K=\mathbf{Q}$ and $L$ is imaginary, and $b=2$ otherwise. Moreover, the index $\left[E_{L}: E_{M} E_{M^{\prime}} E_{N}\right]=p^{a}$ with $0 \leqq a \leqq b$. Furthermore, if $L / N$ is unramified, then $a=b-1$, that is,

$$
h(L)=\frac{1}{p} \cdot \frac{h(M)^{2} h(N)}{h(K)^{2}} .
$$

Let $K$ be an imaginary quadratic number field whose $p$-class group is cyclic of order $p$. Then the Hilbert $p$-class field of $K$ is a $D_{p}$-extension of $\mathbf{Q}$. If $p=3$, or 5 , by Lemma 3 we can compute its class number by calculating the class number of its subfield of degree $p$. For this purpose, we use data for cubic fields and quintic fields. Data for number fields of degrees $n$ with $3 \leqq n \leqq 7$ and small discriminant (in absolute value) are available by anonymous ftp from megrez.math.u-bordeaux.fr (147.210.16.17).

LEMMA 4. ([9]) Let $L$ be an imaginary $D_{4}$-extension of Q. Let $M$ and $N$ be the two nonisomorphic nonnormal quartic subfields and $K$ its quadratic subfield such that $L / K$ is cyclic. Then we have the following class number relation:

$$
h(L)=\frac{\left[E_{L}: E_{M} E_{N} E_{K}\right]}{2^{v}} \cdot h(M) h(N) h(K) .
$$

Here, $v=3$ if $L$ is a CM-field and $v=2$ otherwise. Moreover, the index $\left[E_{L}: E_{M} E_{N} E_{K}\right]$ is a nonnegative power of 2 .

If $K$ is an imaginary quadratic number field with cyclic 2-class group of order $\geqq 4$, then by this lemma the class number of the unique unramified cyclic quartic extension $L$ of $K$ can be computed from the class numbers of two nonnormal quartic subfields of $L$. In this case, $L$ is a $D_{4}$-extension of $\mathbf{Q}$ 
and all of its nonnormal quartic subfields have odd class number. Therefore we have

$$
h(L)=h(M) h(N) h(K) / 4
$$

because the exact 2-power dividing $h(L)$ is $h^{(2)}(K) / 4$, where $h^{(2)}(K)$ is the 2-class number of $K$.

\section{ThE ACtion OF GALOIS GROUPS ON CLASS GROUPS}

The action of Galois groups on class groups can often be used to obtain useful information on the structure of class groups.

We first review the following, often called $p$-rank theorem.

LEMma 5. (See [53, Theorem 10.8]) Let $L / K$ be a finite cyclic extension of degree $n$ of algebraic number fields. Let $p$ be a prime number with $p \nmid n$ and assume that all fields $E$ with $K \subseteq E \varsubsetneqq L$ have trivial $p$-class group. Then for each positive integer $a$, the $\bar{p}^{a}$-rank of the $p$-class group of $L$ is a multiple of the order $f$ of $p$ modulo $n$. In particlar, if $p \mid h(L)$, then $p^{f} \mid h(L)$, and if $h(L)<p^{f}$, then $p \nmid h(L)$.

The following proposition gives us more information in a sense.

Proposition 2. Let $K$ be an imaginary abelian number field and $K_{g}$ its genus field, that is, the maximal unramified abelian extension of $K$ which is abelian over $\mathbf{Q}$. Let $L$ be the Hilbert class field of $K_{g}$. Then for any prime number $p$ with $p \nmid[L: \mathrm{Q}]$, the $p$-class group $\mathrm{Cl}^{(p)}(L)$ of $L$ is trivial or noncyclic.

Proof. We first note that both $L$ and its Hilbert $p$-class field $M$ are normal over $\mathbf{Q}$. The Galois group $\Gamma=\operatorname{Gal}(L / \mathbf{Q})$ acts by conjugation on $\operatorname{Gal}(M / L)$, which is isomorphic to $\mathrm{Cl}^{(p)}(L)$ by class field theory. This action induces a group homomorphism

$$
\rho: \Gamma \rightarrow \operatorname{Aut}\left(\mathrm{Cl}^{(p)}(L)\right)
$$

Now we assume that $\mathrm{Cl}^{(p)}(L)$ is cyclic. Then $\operatorname{Aut}\left(\mathrm{Cl}^{(p)}(L)\right)$ is abelian and therefore the kernel of $\rho$ contains the derived group of $\Gamma$. Hence if we let $F$ be the field corresponding to $\operatorname{Ker}(\rho), F$ is abelian over $\mathbf{Q}$ and therefore $F$ is contained in $K_{g}$. Since $\operatorname{Ker}(\rho)=\operatorname{Gal}(L / F)$ acts on $\operatorname{Gal}(M / L)$ trivially, by the assumption $p \nmid[L: \mathbf{Q}]=|\Gamma|$ we conclude that $\operatorname{Gal}(M / F)$ is factored as

$$
\operatorname{Gal}(M / F)=\operatorname{Gal}(M / L) \times H, \text { with } H \cong \operatorname{Ker}(\rho)
$$


This implies that the class number of $F$ is divisible by $|\operatorname{Gal}(M / L)|=$ $\left|\mathrm{Cl}^{(p)}(L)\right|$ and therefore $\left|\mathrm{Cl}^{(p)}(L)\right| \mid h\left(K_{g}\right)$. Hence $\mathrm{Cl}^{(p)}(L)$ must be trivial.

Remark. As described in the Introduction, if $K$ is an imaginary quadratic number field, we can easily calculate the class number of $K_{g}$. Thus, this will be a powerful tool.

The group homomorphism $\rho$ above often gives a useful information on $\mathrm{Cl}^{(l)}(L)$ also for prime divisors $l$ of $[L: \mathbf{Q}]$. The same argument works in more general situation. The referee pointed out that this proposition is a special case of a result due to $O$. Grün with proofs (essentially the same as above) by L. Holzer and A. Scholz (Jaresber. DMV 44 (1934), 74-75).

Let $L / K$ be a finite Galois extension of algebraic number fields with Galois group $\Gamma$. Let $p$ be a prime number and $A$ the $p$-class group of $L$. The action of $\Gamma$ on $V=A^{p^{a-1}} / A^{p^{a}}$ induces a group homomorphism

$$
\Gamma \rightarrow \operatorname{Aut}(V) \cong \mathrm{GL}(r, p),
$$

where $r$ is the $p^{a}$-rank of $A$. Thus, the knowledge of the structure of linear groups over finite fields is often useful for the study of the structure of class groups. Later we use the following facts.

Lemma 6. ([22, II, Satz 7.3a]) Let $p$ be a prime and $f$ and $n$ positive integers. $\mathrm{GL}\left(f, p^{n}\right)$ has a cyclic subgroup $S$ of order $p^{f n}-1$. ( $S$ is called Singer cycle.) For any subgroup $T$ of $S$ whose order is not a divisor of $p^{e n}-1$ for any $e<f$, its normalizer in $\mathrm{GL}\left(f, p^{n}\right)$ is the semi-direct product of $S$ with a cyclic group $C$ of order $f$ such that the action of one generator of $C$ on $S$ is $p^{n}$ th powering.

Remark. Let $q$ be a prime number $\neq p$ and $f$ the order of $p$ modulo $q$. Then any Sylow $q$-subgroup of GL $(f, p)$ is a subgroup of a Singer cycle and its normalizer in $\mathrm{GL}(f, p)$ has order $f\left(p^{f}-1\right)$.

LEMMA 7. ([8]) Let $q$ be a power of an odd prime and $W$ a Sylow 2-subgroup of $\mathrm{GL}(2, q)$. If $q \equiv 1(\bmod 4)$, then $W$ is isomorphic to $\left.C_{2} s\right\} C_{2}$, the wreath product of $C_{2}$ by $C_{2}$, where $2^{s}$ is the exact power of 2 dividing $q-1$, while if $q \equiv 3(\bmod 4)$, then $W$ is a semi-dihedral group of order $2^{t+2}$, where $2^{t}$ is the exact power of 2 dividing $q+1$.

Remark. By this lemma Sylow 2-subgroups of $\operatorname{GL}(2,3)$ and $\operatorname{GL}(2,5)$ are isomorphic to $S D_{16}$ and $C_{4} / C_{2}\left(=32 \Gamma_{3} e\right)$, respectively. We note that 
$C_{4} \prec C_{2}$ has three maximal subgroups isomorphic to $C_{4}^{2}, D_{4} \curlyvee C_{4}$, and $S D_{16}$. Here, $D_{4} \curlyvee C_{4}$ denotes the central product of $D_{4}$ and $C_{4}$.

Later for an unramified Galois extension $L$ of an imaginary quadratic number field which is normal over $\mathbf{Q}$, we consider the action of the $\mathrm{Ga}$ lois group $\operatorname{Gal}(L / Q)$ on the class group $\mathrm{Cl}(L)$ of $L$. Then an important problem is to determine the image of the induced group homomorphism $\operatorname{Gal}(L / \mathbf{Q}) \rightarrow \operatorname{Aut}(\mathrm{Cl}(L))$. Thus, we prepare the following.

LEMMA 8. Let $K$ be a quadratic number field and $L$ its finite extension which is normal over $\mathbf{Q}$. Suppose that $L / K$ is unramified at all finite primes. Then the Galois group of $L / Q$ is generated by elements of order two not contained in the Galois group of $L / K$. In particular, Gal $(L / Q)$ is isomorphic to none of the 2-groups $C_{4}, Q_{8}, S D_{16}$, and $C_{4} / C_{2}$.

The former assertion of this is a special case of [11, Corollary 16.31], which is easily deduced from Čebotarev monodromy theorem. None of the 2-groups $C_{4}, Q_{8}, S D_{16}$, and $C_{4} / C_{2}$ is generated by elements of order two. We note that if a quadratic number field has an unramified quaternionic extension which is normal over $\mathbf{Q}$, then its Galois group over $\mathbf{Q}$ is isomorphic to $D_{4} \curlyvee C_{4}([30])$.

\section{RESULTS ON CLASS FIELD TOWER}

We also use results on class field tower. We review here some results. The following is well known.

Lemma 9. (See [49, Theorem I].) Let $K$ be an algebraic number field of finite degree and $p$ any prime number. If the $p$-class group, i.e., the p-part of the class group of $K$ is cyclic, then the p-class group of the Hilbert $p$ class field of $K$ is trivial. Moreover, if $p=2$ and the 2-class group of $K$ is isomorphic to $V_{4}$, then the 2-class group of the second Hilbert 2-class field, that is, the Hilbert 2-class field of the Hilbert 2-class field, of $K$ is trivial.

This can be easily proved by using group theory.

All fields we consider have cyclic $p$-class group for any odd prime $p$. In fact, a noncyclic $p$-class group for an odd prime $p$ first occurs as $\mathrm{Cl}(\mathrm{Q}(\sqrt{-3299}))$, which is isomorphic to $C_{9} \times C_{3}([7])$. On the other hand, noncyclic 2-class groups often occur as the 2-class group of $\mathrm{Q}(\sqrt{d}), d<0$ with small $|d|$. But for all fields we consider, the 4-ranks of the class groups are at most one. The first example for bigger 4-rank is $d=-2379$. 
In the rest of this section, we review some results on the 2-class field towers of imaginary quadratic number fields $K$ :

$K=K_{0}^{(2)} \subseteq K_{1}^{(2)} \subseteq K_{2}^{(2)} \subseteq \cdots\left(K_{i+1}^{(2)}\right.$ is the Hilbert 2-class field of $K_{i}^{(2)}$.)

By the lemma above, if the 2-class group $\mathrm{Cl}^{(2)}(K)$ of $K$ is cyclic, then the 2-class field tower of $K$ terminates with $K_{1}^{(2)}$, that is, $K_{2}^{(2)}=K_{1}^{(2)}$. When does $K_{2}^{(2)}=K_{1}^{(2)}$ hold? F. Lemmermeyer [29] gave a necessary condition: If $K_{2}^{(2)}=K_{1}^{(2)}$, then $\mathrm{Cl}^{(2)}(K)$ is cyclic, or isomorphic to $C_{2} \times C_{2^{m}}(m \geqq 1)$. He also obtained sufficient criteria in terms of the factorization of $d$.

If $\mathrm{Cl}^{(2)}(K) \cong V_{4}$, then $K_{3}^{(2)}=K_{2}^{(2)}$ by the lemma above. We know that any nonabelian finite groups of order $2^{n}$ with $n>2$ whose abelianization is isomorphic to $V_{4}$ is isomorphic to $D_{2^{n-1}}, Q_{2^{n}}$, or $S D_{2^{n}}$. Thus, if $\mathrm{Cl}^{(2)}(K) \cong$ $V_{4}$, then $\operatorname{Gal}\left(K_{2}^{(2)} / K\right) \cong V_{4}, D_{2^{n-1}}, Q_{2^{n}}$, or $S D_{2^{n}}$, where $n$ is determined by $2^{n-2} \| h\left(K_{1}^{(2)}\right)$ (and therefore $n$ can be easily calculated, because $K_{1}^{(2)}=$ $K_{g}$ ). H. Kisilevsky [25] characterized the occurrence of these groups in terms of the factorization of the discriminant $d$ of $K$.

When does $K_{3}^{(2)}=K_{2}^{(2)}$ hold? The complete answer has not been given yet. However, Lemmermeyer [29] determined when $\operatorname{Gal}\left(K_{2}^{(2)} / K\right)$ is metacyclic. (Of course, if $\operatorname{Gal}\left(K_{2}^{(2)} / K\right)$ is metacyclic, $K_{3}^{(2)}=K_{2}^{(2)}$ by Lemma 9.) He proved that if $\operatorname{Gal}\left(K_{2}^{(2)} / K\right)$ is nonabelian but metacyclic, then $\mathrm{Cl}^{(2)}(K) \cong V_{4}$, or $d$ is of the form $-4 p q$, where $p$ and $q$ are primes congruent to 5 modulo 8:

(i) If $(p / q)=1$, then $\operatorname{Gal}\left(K_{2}^{(2)} / K\right)$ is isomorphic to the group given by

$$
\left\langle\sigma, \tau \mid \tau^{2^{n+1}}=1, \sigma^{4}=\tau^{2^{n}}, \sigma^{-1} \tau \sigma=\tau^{-1}\right\rangle,
$$

or

$$
\left\langle\rho, \sigma \mid \rho^{2^{n+1}}=\sigma^{2^{m}}=1, \sigma^{-1} \rho \sigma=\rho^{-1}\right\rangle,
$$

according as the norm of the fundamental unit of the real quadratic number field $\mathbf{Q}(\sqrt{p q})$ is -1 or 1 . Here, $n$ is determined by $2^{n} \| h(\mathbf{Q}(\sqrt{p q})$ ) (in both cases), and $m$ is determined by $2^{m+n} \| h(\mathbf{Q}(\sqrt{-1}, \sqrt{p q}))$. In [5], these groups are denoted by $M C_{n}^{-}$and $M C_{n, m}^{+}$, respectively. We use these notations in the Appendix 2.

(ii) If $(p / q)=-1$, then $\operatorname{Gal}\left(K_{2}^{(2)} / K\right) \cong M_{2^{m+2}}$, where $m$ is determined by $2^{m+1} \| h(K)$. Note that the presentation of $\operatorname{Gal}\left(K_{2}^{(2)} / K\right)$ given in [29] is

$$
\left\langle\rho, \sigma \mid \rho^{2}=\sigma^{2^{m}}, \rho^{4}=1, \sigma^{-1} \rho \sigma=\rho^{-1}\right\rangle
$$




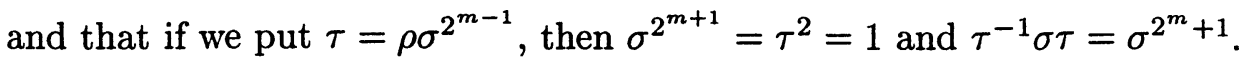

Recently E. Benjamin, F. Lemmermeyer, and C. Snyder characterized $K$ whose 2-class fields have cyclic 2-class group [5]. They proved that $\operatorname{Gal}\left(K_{2}^{(2)} / K\right)$ is nonmetacyclic and its derived group is cyclic, if and only if $d$ is of one of the following forms: $d=-4 p p^{\prime}$, where $p$ and $p^{\prime}$ are primes with $p \equiv 1, p^{\prime} \equiv 5(\bmod 8)$ and $\left(p / p^{\prime}\right)=1,\left(p / p^{\prime}\right)_{4}\left(p^{\prime} / p\right)_{4}=-1 ; d=$ $-r p p^{\prime}$, where $-r$ is a negative discriminant $\neq-4$, and $p$ and $p^{\prime}$ are positive prime discriminants with $\left(p / p^{\prime}\right)=(r / p)=1$ and $\left(r / p^{\prime}\right)=\left(p / p^{\prime}\right)_{4}\left(p^{\prime} / p\right)_{4}=$ -1. Moreover, $\operatorname{Gal}\left(K_{2}^{(2)} / K\right)$ is isomorphic to $\Gamma_{m, t}^{1}$ or $\Gamma_{m, t}^{2}$, according as $d=-4 p p^{\prime}$ (in this case $2^{t}=h^{(2)}\left(\mathbf{Q}(\sqrt{-4 p})\right.$ ), or $d=-r p p^{\prime}$ (in this case $2^{t}=h^{(2)}(\mathbf{Q}(\sqrt{-r p}))$, and in both cases $2^{m}=h^{(2)}(K) / 2$.

\section{Determination of $\operatorname{Gal}\left(K_{u r} / K\right)$}

In this section, we determine $\operatorname{Gal}\left(K_{u r} / K\right)$. Our procedure for each $K=$ $\mathrm{Q}(\sqrt{d})$ is as follows:

(1) We take a normal unramified extension $L$ of $K$ of large degree for which we can obtain an upper bound for $\left[K_{u r}: L\right]$ less than 60 by discriminant bounds (unconditional for $|d| \leqq 420$ and conditional for $|d|>420$ ).

(2) We show $h(L)=1$ and conclude $K_{u r}=L$ by Proposition 1. (For fields with cyclic class group of odd order prime to 15 , we can conclude $K_{u r}=K_{1}$ from $\left[K_{u r}: K_{1}\right]<168$ (see below).)

(3) We determine the structure of $\operatorname{Gal}\left(K_{u r} / K\right)$. (This is not difficult for most fields.)

As described in $\S 2$, for most $K$, we take $L=\left(K_{g}\right)_{1}$. The exceptional cases are the following two cases:

(E1) $d$ is divisible by the discriminant $d_{E}$ of a quartic number field $E$ and the quotient $d / d_{E}$ is a fundamental quadratic discriminant or 1 . In this case the normal closure $M$ of $E$ is an unramified $A_{4}$-extension of the quadratic number field $\mathbf{Q}\left(\sqrt{d_{E}}\right)$ and the composite field $K M$ is not contained in $\left(K_{g}\right)_{1}$. This case is divided into the following three subcases:

(a) $d=d_{E}=-p$, where $p$ is a prime $\equiv 3(\bmod 4): d=-283,-331,-491$, $-563,-643,-751$. In this case, $K$ has an unramified $\widetilde{A_{4}}$-extension, which yields a quaternionic extension of $K_{1}=\left(K_{g}\right)_{1}$ by composition.

(b) $d=d_{E}$ is a composite: $d=-731=17 \cdot(-43)$. In this case, $K$ has an unramified $A_{4}$-extension, which yields a $V_{4}$-extension of $K_{1}=\left(K_{g}\right)_{1}$ by composition.

(c) $d=d^{\prime} d_{E}$, where $d^{\prime}$ is a fundamental quadratic prime discriminant: 
$d=-687=(-3) \cdot 229, d=-771=(-3) \cdot 257, d=-916=(-4) \cdot 229$. In this case, $K$ has an unramified $S_{4}$-extension, which yields a $V_{4}$-extension of $\left(K_{g}\right)_{1}$ by composition.

(E2) Though $K$ does not satisfy the condition in (E1), we can check that $K$ has an unramified $S_{4}$-extension: $d=-856,-996$.

These exceptional cases will be treated in the next section. In the rest of this section, we treat the other fields. Let $L=\left(K_{g}\right)_{1}$. Then we will show $K_{u r}=L=\left(K_{g}\right)_{1}$.

We can treat fields with same class group similarly. (Of course, for some fields, additional consideration is needed.) None of $K$ with $h(K)=1$ has any nontrivial unramified extension. For the fields $K$ with $h(K)=2$, we have determined $\operatorname{Gal}\left(K_{u r} / K\right)$ in [58].

We first treat fields with odd class number $\geqq 3$. Note that $K_{g}=$ $K$ if and only if $h(K)$ is odd. We note that except for fields listed in (a) no fields with odd class number appear in our table as fields with $l \geqq 2$. For all $K$ with odd class number and $|d|<1000$ except for $d=$ $-787,-827,-859,-883,-947,-967,-971,-991$, we get $\left[K_{u r}: K_{1}\right]<60$ by discriminant bounds. Therefore our task is to prove $h\left(K_{1}\right)=1$. We have the following.

Proposition 3. Let $K$ be an imaginary quadratic number field with cyclic class group of odd order $n \geqq 7$. Assume that for all intermediate fields $E$ of $K_{1} / K$, we have $E_{1}=K_{1}$. Then if $h\left(K_{1}\right)>1$ (this is equivalent to $K_{2} \neq K_{1}$ ), we have $h\left(K_{1}\right) \geqq 2^{6}=64$. (This is valid also for real quadratic number fields. In the case where $K$ is imaginary and $h(K)$ is a prime $(\geqq 7)$, $h\left(K_{1}\right) \geqq 64$ can be improved to $h\left(K_{1}\right) \geqq 13^{2}=169$. See the remark after the proof.)

Proof. Let $l$ be a prime factor of $h(K)=n$. Then by the assumption there exists a subextension $E$ of $K_{1} / K$ such that $h(E)=l$ and $E_{1}=K_{1}$. Therefore $l \nmid h\left(K_{1}\right)$ by Lemma 9 .

Let $p$ be a prime factor of $h\left(K_{1}\right)$ and $r$ the $p$-rank of the $p$-class group of $K_{1}$. We will show $p^{r} \geqq 2^{6}=64$. Since by Lemma 5 and Proposition 2 we have $r \geqq 2$ and $p^{r} \equiv 1(\bmod n)$, it suffices to eliminate the following four possibilities: (a) $p=2, r=3$ if $n=7$; (b) $p=2, r=4$ if $n=15$; (c) $p=2, r=5$ if $n=31$; (d) $p=3, r=3$ if $n=13$. Note that for all these possibilities, $r$ is the order of $p \bmod n$.

Put $\Gamma=\operatorname{Gal}\left(K_{1} / \mathrm{Q}\right) \cong D_{n}$ and $\Lambda=\operatorname{Gal}\left(K_{1} / K\right) \cong C_{n}$. The action of $\Gamma$ on $\mathrm{Cl}\left(K_{1}\right) / \mathrm{Cl}\left(K_{1}\right)^{p} \cong \mathbf{F}_{p}^{r}$ induces a group homomorphism

$$
\Gamma \rightarrow \mathrm{GL}(r, p) \text {. }
$$


If $\Lambda \cap \operatorname{Ker}(\rho) \neq\{1\}$, then we would have $p^{r} \mid h(F)$ for the field $F$ corresponding to $\Lambda \cap \operatorname{Ker}(\rho)$, which contradicts the assumption. (Cf. the proof of Proposition 2.) Therefore $\Lambda \cap \operatorname{Ker}(\rho)=\{1\}$ and $\rho(\Lambda) \cong \Lambda \cong C_{n}$. Since $\operatorname{Im}(\rho)$ is a factor group of $\Gamma \cong D_{n}, \operatorname{Im}(\rho) \cong D_{n}$. Since $\Lambda$ is a normal subgroup of $\Gamma, \operatorname{Im}(\rho)$ is contained in the normalizer $N$ of $\rho(\Lambda)$ in $\operatorname{GL}(r, p)$. Therefore $N$ must have a subgroup of isomorphic to $D_{n}$.

Assume that $n$ is a prime and that $r$ is the order of $p \bmod n$. Then $\rho(\Lambda)$ is a subgroup of a Singer cycle of GL $(r, p)$ satisfying the condition of Lemma 6. Therefore $N \cong C_{p^{r}-1} \rtimes C_{r}$. Since $N$ has a subgorup of isomorphic to $D_{n}, r$ must be even. This eliminates the posiibilities (a), (c), and (d).

We know GL $(4,2)=\operatorname{PSL}(4,2) \cong A_{8}[22$, II, Satz 6.14, (5)]. Since this group does not have a subgroup isomorphic to $D_{15}, r \neq 4$ if $p=2$ and $n=15$. This completes the proof.

Remark. Regrettably the same argument does not work for $h(K)=5$ : The Singer cycles of $\mathrm{GL}(4,2) \cong A_{8}$ have a subgroup isomorphic to $D_{5}$. However, the class number relation (Lemma 3) enables us to compute $h\left(K_{1}\right)$ by calculating class numbers of quintic subfields of $K_{1}$. For all $K$ with $h(K)=5($ all such $K$ have discriminant $\leqq 2683[2,52])$, we have $h\left(K_{1}\right)=1$ and therefore $K_{2}=K_{1}$. We note that we can find in [20] the class numbers of the unramified cyclic quintic extensions $F$ of $K$ with $5 \mid h(K)$ and $|d|<1000$. For all such $K, h(F)=h(K) / 5$. By Lemma 3 we can check that for all $K$ with $3 \mid h(K)$ and $|d|<1000$ except for $d$ listed in (a), we have $h(F)=h(K) / 3$, where $F$ is the unique unramified cyclic cubic extension of $K$.

F. Hajir checked the parities of $h\left(K_{1}\right)$ of $K$ with $\mathrm{Cl}(K) \cong C_{q}(q$ an odd prime $\leqq 19)$ and $|d|<15000$ by using elliptic units [16]: For all such $K$ except for $d=-283(q=3),-331(q=3),-643(q=3)$, and -14947 $(q=17), h\left(K_{1}\right)$ is odd $\left(2^{8}=256 \mid h\left(K_{1}\right)\right.$ for $\left.d=-14947\right)$. All $K$ with $h(K)=7$ have discriminant $\leqq 5923[2,52]$ and therefore $h\left(K_{1}\right)$ is odd for all such $K$. By this reason in the case where ( $K$ is imaginary and) $h(K)=q$ ( $q$ an odd prime $\geqq 7), h\left(K_{1}\right) \geqq 64$ can be improved to $h\left(K_{1}\right) \geqq 13^{2}=169$. (Note that $\mathrm{GL}(2,13)$ has a subgroup isomorphic to $D_{7}$.) Moreover, in the case where $h(K) \geqq 11$ and $h(K) \neq 21,63, h\left(K_{1}\right) \geqq 64$ can be improved to $h\left(K_{1}\right) \geqq 2^{8}=256$ (without assuming the imaginarity of $K$.) (Note that $\mathrm{GL}(8,2)$ has a subgroup isomorphic to $D_{17}$.)

Suppose $\mathrm{Cl}(K) \cong C_{n}$, where $n$ is an odd integer prime to 15 . The estimate $h\left(K_{1}\right) \geqq 169$ under the assumption $h\left(K_{1}\right)>1$ is important. By this, if $\left[K_{u r}: K_{1}\right]<168$, then we can conclude $K_{u r}=K_{1}$ under the assumption that $K$ does not have an unramified $A_{5}$-extension which is 
normal over $\mathbf{Q}$. (Though there exist (probably infinitely) many imaginary quadratic number fields with cyclic class group of odd order prime to 15 having such an extension, the maximal discriminant of such fields is -2083 . $(h(\mathbf{Q}(\sqrt{-2083})=7$. See $\S 8$. $))$ For this conclusion, we need only to show that $K_{1}$ does not have an unramified $A_{5}$-extension. (Note that the second minimal order of nonabelian simple groups is 168.) Suppose that $K_{1}$ has such an extension $M$. Then the Galois group $\operatorname{Gal}(M / K)$ is an extension of $A_{5}$ by $C_{n}$. Since any such extension is the direct product $A_{5} \times C_{n}, K$ has an unramified $A_{5}$-extension and this extension must be normal over $\mathbf{Q}$, for otherwise its normal closure has degree $2 \cdot 60^{2}=7200$. This contradicts the assumption. Thus, $\left[K_{u r}: K_{1}\right]<168$ implies $K_{u r}=K_{1}$. For $K=$ $\mathbf{Q}(\sqrt{-827}), \mathbf{Q}(\sqrt{-859}), \mathbf{Q}(\sqrt{-967}), \mathbf{Q}(\sqrt{-991})$, we do not get $\left[K_{u r}: K_{1}\right]<$ 60 but get $\left[K_{u r}: K_{1}\right]<168$ by Odlyzko's (conditional) discriminant bounds and therefore $K_{u r}=K_{1}$ (under GRH).

Now we treat fields $K$ with even class number. For such $K, K_{g} \neq K$. First we calculate $h\left(K_{g}\right)$. We have two cases:

(i) $h\left(K_{g}\right)=h(K) /\left[K_{g}: K\right]$.

(ii) $h\left(K_{g}\right)>h(K) /\left[K_{g}: K\right]$.

The equality $h\left(K_{g}\right)=h(K) /\left[K_{g}: K\right]$ is equivalent to $\left(K_{g}\right)_{1}=K_{1}$. (This holds trivially in the case of odd class number.) If $K_{u r}=K_{1}$, then we have $\left(K_{g}\right)_{1}=K_{1}$. We expect the converse, that is, if $\left(K_{g}\right)_{1}=K_{1}$, then $K_{u r}=K_{1}$ holds for fields considered. In fact, we can show this for most $K$.

We first treat fields with nontrivial cyclic 2-class group. For such $K$, its discriminant $d$ is the product of two prime discriminants: $d=d_{1} d_{2}$ and $K_{g}=\mathbf{Q}\left(\sqrt{d_{1}}, \sqrt{d_{2}}\right)$. Since $\frac{1}{2} h(K) \mid h\left(K_{g}\right)$ but $h(K) \nmid h\left(K_{g}\right)$ by Lemma 9 , we have $h\left(K_{g}\right)=h(K) h\left(\mathbf{Q}\left(\sqrt{d_{1}}\right)\right) h\left(\mathbf{Q}\left(\sqrt{d_{2}}\right)\right) / 2$ by Lemma 2 . (Note that since $d_{1}$ and $d_{2}$ are prime discriminants, both $h\left(\mathbf{Q}\left(\sqrt{d_{1}}\right)\right)$ and $h\left(\mathbf{Q}\left(\sqrt{d_{2}}\right)\right)$ are odd. More precisely, we have

$$
\mathrm{Cl}\left(K_{g}\right) \cong \mathrm{Cl}(K)^{2} \times \mathrm{Cl}\left(\mathbf{Q}\left(\sqrt{d_{1}}\right)\right) \times \mathrm{Cl}\left(\mathbf{Q}\left(\sqrt{d_{2}}\right)\right),
$$

where $\mathrm{Cl}(K)^{2} \neq \mathrm{Cl}(K) \times \mathrm{Cl}(K)$ but $\mathrm{Cl}(K)^{2}=\left\{c^{2} \mid c \in \mathrm{Cl}(K)\right\}$, because the odd part of the class group of a biquadratic bicyclic number field is isomorphic to the direct product of those of its quadratic subfields (see [28]). Hence in this case $h\left(K_{g}\right)=h(K) /\left[K_{g}: K\right]$ is equivalent to $h\left(\mathbf{Q}\left(\sqrt{d_{1}}\right)\right)=h\left(\mathbf{Q}\left(\sqrt{d_{2}}\right)\right)=1$. We take $d_{2}$ as $d_{2}>0$. How often does $h\left(\mathbf{Q}\left(\sqrt{d_{2}}\right)\right)=1$ occur? H. Cohen and H. W. Lenstra, Jr. [10] posed heuristic conjectures (the so-called Cohen-Lenstra heuristics) on the distribution of class groups of algebraic number fields. Their conjectures state that the 
probability that a real quadratic number field with prime discriminant has class number one is about 0.75446 and this has been numerically supported by A. G. Stephens and H. C. Williams [48]. The first two discriminants of real quadratic number fields with prime discriminant and class number $>1$ are 229 and 257. These are also discriminants of quartic number fields! The Cohen-Lenstra heuristics state also that the probability that an imaginary quadratic number field has class group whose odd part is cyclic is about 0.97757. Thus, for simplicity, we consider here fields with $h\left(\mathbf{Q}\left(\sqrt{d_{2}}\right)\right)=1$ and $\mathrm{Cl}\left(\mathrm{Q}\left(\sqrt{d_{1}}\right)\right) \cong C_{n}(n$ an odd integer $)$. Then $h\left(K_{g}\right)=h(K) /\left[K_{g}: K\right]$ is equivalent to $n=1$. When $n=1$, under some condition by considering Galois action as in Proposition 3, we get an estimate for $h\left(K_{1}\right)$. When $n>1$, we can easily determine the structure of $\operatorname{Gal}\left(\left(K_{g}\right)_{1} / K\right)$. We first give an estimate for $h\left(K_{1}\right)$ if $n=1$ and then determine $\operatorname{Gal}\left(\left(K_{g}\right)_{1} / K\right)$ if $n>1$.

Proposition 4. Let $K$ be an imaginary quadratic number field with nontrivial cyclic 2-class group.

(i) Assume $\mathrm{Cl}(K) \cong C_{2^{m}}(m \geqq 3)$ and that the unique unramified cyclic quartic extension $E$ of $K$ has class number $2^{m-2}$. (The latter assumption implies $h\left(K_{g}\right)=h(K) / 2$.) If $h\left(K_{1}\right)>1$, then we have $h\left(K_{1}\right) \geqq 7^{2}=49$.

(ii) Assume $h(K)=2 q(q$ an odd prime $\geqq 7)$ and $h\left(K_{g}\right)=q(=h(K) / 2)$. If $h\left(K_{1}\right)>1$, then we have $h\left(K_{1}\right) \geqq 2^{6}=64$.

(These are valid also for real quadratic number fields if $K_{g}$ is the unique unramified quadratic extension of $K$.)

Proof. We give only a sketch.

(i) Put $\Gamma=\operatorname{Gal}\left(K_{1} / \mathrm{Q}\right)$. Then $\Gamma \cong D_{2^{m}}$. By Lemmas 7 and 8 any group homomorphisms $\Gamma \rightarrow \mathrm{GL}(2,3)$ and $\Gamma \rightarrow \mathrm{GL}(2,5)$ have image of order at most eight and this implies that neither $\mathrm{Cl}\left(K_{1}\right) \cong C_{3}^{2}$ nor $\mathrm{Cl}\left(K_{1}\right) \cong C_{5}^{2}$ can occur by the assumption. (Cf. the proof of Proposition 2. Note that the Sylow 2-subgroups of $\mathrm{GL}(2,7)$ are isomorphic to $S D_{32}$ and they have a subgroup isomorphic to $D_{8}$.) Hence we get the conclusion.

(ii) Put $F=\mathrm{Q}\left(\sqrt{d_{1}}\right)$. Then $\operatorname{Gal}\left(K_{1} / F\right) \cong D_{q}$. Thus, the same argument as in the proof of Proposition 3 works.

Remark. Note that when $K$ has an unramified cyclic quartic extension $E$, we can calculate $h(E)$ by using Lemma 4 . The fields with $\mathrm{Cl}(K) \cong$ $C_{2^{m}}(m \geqq 2)$ and $|d|<1000$ are divided into two types: fields $K$ with $h(E)=2^{m-2}$ and fields $K$ with $h\left(K_{g}\right)=2^{m-1} q$ ( $q$ an odd prime). For most of the fields of the former type, we can easily check $K_{u r}=K_{1}$ by using the proposition above. For the fields of the latter type, we can check $K_{u r}=\left(K_{g}\right)_{1}$. Then we consider the Galois group. 
Also when $K$ has an unramified cyclic octic extension $F$, we can calculate $h(F)$ by using Lemma 2 , however we must calculate the factorization of a rational prime in its nonnormal quartic subfield and find a suitable generator of a prime factor. (For the construction of $F$, see [54].) For example, let $K=\mathrm{Q}(\sqrt{-904})$. Then $\mathrm{Cl}(K) \cong C_{8}$. First we calculate the class number of the unique unramified cyclic quartic extension $E$ of $K$. Its nonisomorphic nonnormal subfields are $\mathbf{Q}(\sqrt{9+4 \sqrt{-2}})$ and $\mathbf{Q}(\sqrt{(9+\sqrt{113}) / 2})$. Both of them have class number one and therefore $h(E)=2$. Put $\alpha=\sqrt{9+4 \sqrt{-2}}$ and $N=\mathbf{Q}(\alpha)$. Then $K_{1}$ is a $V_{4}$-extension of $N$. To obtain $h\left(K_{1}\right)$, we calculate the class number of an intermediate field $(\neq E)$ of $K_{1} / N$. By using KANT we get the factorization of the rational prime 113 in the ring of integers of $N$ and calculate a generator of each prime factor. Then as such an intermediate field we can take $N(\sqrt{\beta})$, where $\beta=104+48 \sqrt{-2}-(235+108 \sqrt{-2}) \alpha$. KANT gives $h(N(\sqrt{\beta}))=1$ and therefore $h\left(K_{1}\right)=1$ by Lemma 2 .

Proposition 5. Let $K$ be an imaginary quadratic number field with cyclic class group of order $2^{m}(m \geqq 2)$. Assume that the class group of its genus field $K_{g}$ is cyclic of order $2^{m-1} n$, where $n$ is an odd integer $\geqq 3$. Then the Galois group of the Hilbert class field $L$ of $K_{g}$ over $K$ is isomorphic to $I_{n}^{2^{m}}$ : $\operatorname{Gal}(L / K) \cong I_{n}^{2^{m}}$.

Proof. In this case, $L$ is a cyclic extension of $K_{1}$ of degree $n$ and therefore $\operatorname{Gal}(L / K)$ is isomorphic to a semi-direct product of $C_{n}$ by $C_{2^{m}}$. Thus, it suffices to show that for each prime factor $p$ of $n$, the action of $\operatorname{Gal}\left(K_{1} / K\right) \cong$ $C_{2^{m}}$ on the Sylow $p$-subgroup of $\operatorname{Gal}\left(L / K_{1}\right)$, which is isomorphic to $\operatorname{Gal}\left(K_{1} F / K_{1}\right) \cong C_{q}$, is given by inversion, that is, $\operatorname{Gal}\left(K_{1} F / K\right) \cong I_{q}^{2^{m}}$, where $F$ is the Hilbert $p$-class field of $K_{g}$ and $q=\left[F: K_{g}\right]$. Since $\operatorname{Gal}\left(K_{1} F / K\right) \cong C_{q} \rtimes C_{2^{m}}$, we can present it as

$$
\left\langle a, b \mid a^{2^{m}}=b^{q}=1, a^{-1} b a=b^{i}\right\rangle
$$

for some $i$ with $q \nmid i$. By the assumption $\mathrm{Cl}\left(K_{g}\right) \cong C_{2^{m-1} n}, a^{2}$ must commute with $b$ and therefore $i= \pm 1$. Since $\operatorname{Gal}\left(K_{1} F / K\right)$ is nonabelian, $i=-1$, that is, $\operatorname{Gal}\left(K_{1} F / K\right) \cong I_{q}^{2^{m}}$.

Remark. All $K$ with $\mathrm{Cl}(K) \cong C_{4}$ satisfy $h\left(K_{1}\right)=1$ or $h\left(K_{g}\right)=2 q(q$ an odd prime). (S. Arno [1] verified that the known list of imaginary quadratic number fields with class number four is complete. Therefore we can easily check this.) Note that $I_{q}^{4} \cong Q_{4 q}$.

Now we consider fields $K=\mathbf{Q}\left(\sqrt{d_{1} d_{2}}\right)$ with $2 \| h(K), h\left(\mathbf{Q}\left(\sqrt{d_{2}}\right)\right)=1$ and $\mathrm{Cl}(F) \cong C_{n}(n$ an odd integer $\geqq 3)$, where $F=\mathbf{Q}\left(\sqrt{d_{1}}\right)$. For such $K$, 
$\mathrm{Cl}\left(K_{g}\right) \cong \mathrm{Cl}_{\text {odd }}(K) \times C_{n}$, where $\mathrm{Cl}_{\text {odd }}(K)$ is the odd part of $\mathrm{Cl}(K)$ and $\operatorname{Gal}\left(K F_{1} / K\right) \cong \operatorname{Gal}\left(F_{1} / \mathrm{Q}\right) \cong D_{n}$. Hence $\operatorname{Gal}\left(\left(K_{g}\right)_{1} / K\right) \cong \operatorname{Gal}\left(K F_{1} / K\right) \times$ $\mathrm{Cl}_{\text {odd }}(K) \cong D_{n} \times \mathrm{Cl}_{\text {odd }}(K)$. Thus, groups of this form occur as $G$.

For the other fields, the 2-rank of the 2-class group is larger than or equal to 2, and the length of the 2-class field tower often becomes two. Therefore an important problem is to determine the Galois group $\operatorname{Gal}\left(K_{2}^{(2)} / K\right)$. For this purpose we do not have sufficient knowledge of the 2-class field tower. In fact, we cannot get the structure of $\operatorname{Gal}\left(K_{2}^{(2)} / K\right)$ for $K=\mathrm{Q}(\sqrt{-660})$ and $K=\mathrm{Q}(\sqrt{-840})$ without additional consideration. For some fields many computer calculations of class numbers are needed. In the rest of this section, we will describe details for selected values of $d$. For simplicity, we denote by $B(2 N)$ the lower bound for the root discriminants of the totally imaginary number fields of (finite) degrees $\geqq 2 N$ instead of $B(2 N, 0, N)$.

$d=-420$. J. Martinet writes in [34] that R. Schoof communicated to him an unconditional proof for $\left[K_{u r}: \mathrm{Q}\right]=64$, but that proof is not given there. The author was informed by Schoof with a complete proof that there had been a gap in the proof he had communicated to Martinet and that this gap was filled by F. Lemmermeyer a few years ago [43]. Recently in [31] he gives an unconditional proof for $\left[K_{u r}: Q\right]=64$. He also determines the structure of $\mathrm{Gal}\left(K_{2}^{(2)} / K\right)$ for some fields $K$ with $\mathrm{Cl}(K) \cong C_{2}^{3}$ from which we get $\operatorname{Gal}\left(K_{u r} / K\right) \cong \Gamma_{1} \cong 32 \Gamma_{4} c_{3}$ for $K=\mathrm{Q}(\sqrt{-420})$, where $\Gamma_{n}$ is the designation used in [31] denoting the group of order $2^{n+4}$ given by

$$
\begin{gathered}
\left.\left.\langle\rho, \sigma, \tau| \rho^{4}=\sigma^{2^{n+1}}=\tau^{4}=1, \rho^{2}={\sigma^{2^{n}} \tau^{2},}^{2}, \tau\right]=1,[\rho, \sigma]=\sigma^{2},[\rho, \tau]=\rho^{2}\right\rangle .
\end{gathered}
$$

We can give another proof for $\operatorname{Gal}\left(K_{u r} / K\right) \cong 32 \Gamma_{4} c_{3}$, but we do not give it here because the situation is similar to the case $K=\mathbf{Q}(\sqrt{-840})$.

$d=-660$. F. Lemmermeyer told the author the structure of $\mathrm{Gal}\left(K_{u r} / K\right)$ and sketch of his proof [32]. We give here another more computational proof.

We have $\mathrm{Cl}(K) \cong C_{2}^{3}, K_{1}=\mathrm{Q}(\sqrt{-1}, \sqrt{-3}, \sqrt{-11}, \sqrt{5})$, and $h\left(K_{1}\right)=8$. We will show $K_{u r}=K_{2}$. Since $r d_{K}=\sqrt{660}=25.6904 \ldots$ and $r d_{K}<B(2$. $8 \cdot 8 \cdot 4)([38])$, we have $\left[K_{u r}: K_{2}\right] \leqq 3$. By Proposition $2, h\left(K_{2}\right) \neq 3$. Before proving $h\left(K_{2}\right) \neq 2$, we determine the structure of $\mathrm{Cl}\left(K_{1}\right)$ and $\mathrm{Gal}\left(K_{2} / K\right)$.

We first show that $\mathrm{Cl}\left(K_{1}\right) \cong C_{4} \times C_{2}$. We have $\mathrm{Cl}(\mathrm{Q}(\sqrt{3}, \sqrt{-55})) \cong$ $C_{8} \times C_{2}$. Therefore $\mathrm{Cl}\left(K_{1}\right)$ contains an element of order at least four. Hence the 2-rank of $\mathrm{Cl}\left(K_{1}\right)$ is at most two, that is, $\mathrm{Cl}\left(K_{1}\right) \cong C_{8}$ or $C_{4} \times C_{2}$. Assume $\mathrm{Cl}\left(K_{1}\right) \cong C_{8}$. Put $G=\operatorname{Gal}\left(K_{2} / K\right)$. Then $G$ is a group of order 
64 such that

$$
G / G^{\prime} \cong C_{2}^{3}, G^{\prime} \cong C_{8}
$$

Therefore $G$ belongs to the family $\Gamma_{8}$ or $\Gamma_{19}$. We note that any quadratic subextensions of $K_{1} / K$ has class number eight or sixteen. In fact, we have

$$
\begin{aligned}
& \mathrm{Cl}(\mathbf{Q}(\sqrt{-1}, \sqrt{165})) \cong \mathrm{Cl}(\mathbf{Q}(\sqrt{-3}, \sqrt{55})) \\
\cong & \mathrm{Cl}(\mathbf{Q}(\sqrt{-5}, \sqrt{33})) \cong \mathrm{Cl}(\mathbf{Q}(\sqrt{11}, \sqrt{-15})) \cong C_{4} \times C_{2}, \\
& \mathrm{Cl}(\mathbf{Q}(\sqrt{5}, \sqrt{-33})) \cong C_{4} \times V_{4}, \operatorname{Cl}(\mathbf{Q}(\sqrt{-11}, \sqrt{15})) \cong C_{2}^{3} \\
& \mathrm{Cl}(\mathbf{Q}(\sqrt{3}, \sqrt{-55})) \cong C_{8} \times C_{2} .
\end{aligned}
$$

Therefore $G$ does not have an abelian subgroup of index two. Hence $G$ must be isomorphic to $64 \Gamma_{19} a_{1}$ or $64 \Gamma_{19} a_{2}$. Now we put $H_{1}=\operatorname{Gal}\left(K_{2} / \mathrm{Q}(\sqrt{3}\right.$, $\sqrt{-55})$ ). Then $H_{1}$ is a group of order 32 such that

$$
H_{1} / H_{1}^{\prime} \cong C_{8} \times C_{2}, H_{1}^{\prime} \cong C_{2}
$$

Therefore $H_{1}$ is isomorphic to $32 \Gamma_{2} j_{1}, 32 \Gamma_{2} j_{2}$, or $32 \Gamma_{2} k$. However, neither $64 \Gamma_{19} a_{1}$ nor $64 \Gamma_{19} a_{2}$ has a subgroup isomorphic to any one of these groups. This is a contradiction. Hence $\mathrm{Cl}\left(K_{1}\right) \cong C_{4} \times C_{2}$.

Next, we determine $G=\operatorname{Gal}\left(K_{2} / K\right)$. $G$ is a group of order 64 such that

$$
G / G^{\prime} \cong C_{2}^{3}, G^{\prime} \cong C_{4} \times C_{2}
$$

Therefore $G$ belongs to the family $\Gamma_{14}, \Gamma_{15}, \Gamma_{16}$, or $\Gamma_{24}$. Since $G$ has a subgroup isomorphic to $32 \Gamma_{2} j_{1}, 32 \Gamma_{2} j_{2}$, or $32 \Gamma_{2} k, G$ belongs to the family $\Gamma_{15}$.

In order to know subgroups of $G$ of order 32 , we determine three such groups $H_{1}, H_{2}=\operatorname{Gal}\left(K_{2} / \mathbf{Q}(\sqrt{5}, \sqrt{-33})\right)$, and $H_{3}=\operatorname{Gal}\left(K_{2} / \mathbf{Q}(\sqrt{-11}\right.$, $\sqrt{15}))$.

First, we determine $H_{1}$ exactly. For this, we determine the class groups of the three intermediate fields of $K_{1}=\mathrm{Q}(\sqrt{-1}, \sqrt{-3}, \sqrt{5}, \sqrt{-11}) / \mathrm{Q}(\sqrt{3}$, $\sqrt{-55})$, that is, of $\mathbf{Q}(\sqrt{-1}, \sqrt{-3}, \sqrt{-55}), \mathbf{Q}(\sqrt{3}, \sqrt{-5}, \sqrt{11})$, and $\mathbf{Q}(\sqrt{3}, \sqrt{5}$, $\sqrt{-11})$. We can execute this by computer. We have

$$
\mathrm{Cl}(\mathrm{Q}(\sqrt{-1}, \sqrt{-3}, \sqrt{-55})) \cong \mathrm{Cl}(\mathbf{Q}(\sqrt{3}, \sqrt{-5}, \sqrt{11})) \cong C_{8} \times C_{2},
$$

and

$$
\mathrm{Cl}(\mathbf{Q}(\sqrt{3}, \sqrt{5}, \sqrt{-11})) \cong C_{4} \times V_{4}
$$


Thus, $H_{1}$ contains two subgroups isomorphic to $C_{8} \times C_{2}$ and one subgroup isomorphic to $C_{4} \times V_{4}$. Hence $H_{1} \cong 32 \Gamma_{2} j_{1}$.

Next, we determine $\mathrm{H}_{2}$. For this, we calculate the class groups of some quadratic subextensions of $K_{1} / \mathrm{Q}(\sqrt{5}, \sqrt{-33})$, that is, of $\mathrm{Q}(\sqrt{3 \pm 2 \sqrt{5}}$, $\sqrt{-33}$ ). (Note that the Hilbert class field of $Q(\sqrt{-55})$ is $\mathbf{Q}(\sqrt{-11}, \sqrt{3+2 \sqrt{5}})=\mathbf{Q}(\sqrt{-11}, \sqrt{3-2 \sqrt{5}})$.) By computer calculation we have

$$
\mathrm{Cl}(\mathrm{Q}(\sqrt{3+2 \sqrt{5}}, \sqrt{-33})) \cong \mathrm{Cl}(\mathrm{Q}(\sqrt{3-2 \sqrt{5}}, \sqrt{-33})) \cong C_{4}^{2} .
$$

Therefore $H_{2}$ contains two subgroups isomorphic to $C_{4}^{2}$ and one subgroup isomorphic to $C_{4} \times V_{4}$. Since $H_{2}$ is a group of order 32 such that

$$
H_{2} / H_{2}^{\prime} \cong C_{4} \times V_{4}, \quad H_{2}^{\prime} \cong C_{2},
$$

we have $H_{2} \cong 32 \Gamma_{2} f$.

Thus, $G$ contains a subgroup isomorphic to $32 \Gamma_{2} j_{1}$ and one isomorphic to $32 \Gamma_{2} f$, and therefore $G \cong 64 \Gamma_{15} f_{1}$ or $64 \Gamma_{15} f_{2}$.

Now, we determine $\mathrm{H}_{3} . \mathrm{H}_{3}$ is a group of order 32 such that

$$
H_{3} / H_{3}^{\prime} \cong C_{2}^{3},\left|H_{3}\right|=4 \text {. }
$$

Since $H_{3}$ is a subgroup of $64 \Gamma_{15} f_{1}$ or $64 \Gamma_{15} f_{2}, H_{3} \cong 32 \Gamma_{4} b_{1}$ or $32 \Gamma_{4} b_{2}$, according as $G \cong 64 \Gamma_{15} f_{1}$ or $64 \Gamma_{15} f_{2}$. To judge the structure of $H_{3}$, we consider its maximal subgroups. Among the seven maximal subgroups of $32 \Gamma_{4} b_{1}$ (resp. $32 \Gamma_{4} b_{2}$ ), three are (isomorphic to) $16 \Gamma_{2} a_{1}$, two are $16 \Gamma_{2} c_{1}$, and one is $16 \Gamma_{2} c_{1}$ (resp. one is $16 \Gamma_{2} a_{2}$, two are $16 \Gamma_{2} c_{1}$, and three are $\left.16 \Gamma_{2} c_{2}\right)$. They correspond to the quadratic subextensions of $K_{2} / \mathbf{Q}(\sqrt{-11}, \sqrt{15})$, that is, $\mathbf{Q}(\sqrt{3}, \sqrt{5}, \sqrt{-11}), \mathbf{Q}(\sqrt{-1}, \sqrt{-11}, \sqrt{-15})$, $\mathbf{Q}(\sqrt{-3}, \sqrt{-5}, \sqrt{-11}), \mathbf{Q}(\sqrt{(3 \pm \sqrt{-11}) / 2}, \sqrt{15}), \mathbf{Q}(\sqrt{-(3 \pm \sqrt{-11}) / 2}, \sqrt{15})$. (Note that the Hilbert class field of $\mathbf{Q}(\sqrt{-55})$ can be expressed as $\mathbf{Q}(\sqrt{5}, \sqrt{(3 \pm \sqrt{-11}) / 2})=\mathbf{Q}(\sqrt{5}, \sqrt{-(3 \pm \sqrt{-11}) / 2})$.) By computer calculation we have

$\mathrm{Cl}(\mathrm{Q}(\sqrt{(3 \pm \sqrt{-11}) / 2}, \sqrt{15})) \cong \mathrm{Cl}(\mathrm{Q}(\sqrt{-(3 \pm \sqrt{-11}) / 2}, \sqrt{15})) \cong C_{4} \times C_{2}$

Noting that both of the abelianizations of $16 \Gamma_{2} a_{1}$ and $16 \Gamma_{2} a_{2}$ (resp. $16 \Gamma_{2} c_{1}$ and $16 \Gamma_{2} c_{2}$ ) are isomorphic to $C_{2}^{3}$ (resp. $C_{4} \times C_{2}$ ), we conclude that $H_{3}$ contains at least four subgroups isomorphic to $16 \Gamma_{2} c_{1}$ or $16 \Gamma_{2} c_{2}$. Therefore $H_{3} \cong 32 \Gamma_{4} b_{2}$. Hence $G \cong 64 \Gamma_{15} f_{2}$. 
Now we prove $h\left(K_{2}\right) \neq 2$. Assume $h\left(K_{2}\right)=2$. If we denote by $\Gamma$ the Galois group $\operatorname{Gal}\left(K_{3} / \mathrm{Q}(\sqrt{-1}, \sqrt{-3}, \sqrt{-55})\right)$. Then $\Gamma$ is a group of order 32 such that

$$
\Gamma / \Gamma^{\prime} \cong C_{8} \times C_{2}, \Gamma^{\prime} \cong C_{2}
$$

Therefore $\Gamma$ is isomorphic to $32 \Gamma_{2} j_{1}, 32 \Gamma_{2} j_{2}$, or $32 \Gamma_{2} k$. All of these groups have only abelian maximal subgroups. This contradicts that the maximal subgroup $\operatorname{Gal}\left(K_{3} / K_{1}\right)$ of $\Gamma$ is nonabelian. Hence $h\left(K_{2}\right) \neq 2$. Thus, $K_{u r}=$ $K_{2}$.

$d=-759$. We have $\mathrm{Cl}(K) \cong C_{12} \times C_{2}, K_{g}=\mathrm{Q}(\sqrt{-3}, \sqrt{-11}, \sqrt{-23})$, and $h\left(K_{g}\right)=18$. Put $L=\left(K_{g}\right)_{1}$. We will show $K_{u r}=L$. Since $r d_{K}=$ $\sqrt{759}=27.5499 \ldots$ and $r d_{K}<B(2 \cdot 4 \cdot 18 \cdot 5)([38])$, we have $\left[K_{u r}: L\right] \leqq 4$. We will show $h(L)=1$.

First we show $h(L) \neq 3$. Put $F=\mathbf{Q}(\sqrt{-23})$. Then $h(F)=3$ and $L=K_{1} F_{1}$. Since $K_{1}^{(2)} / K F$ is cyclic (quartic), $K_{1} / K_{1}^{(3)} F$ is cyclic and therefore $L / K_{1}^{(3)} F_{1}$ is cyclic. Hence if the class number of the intermediate field $K_{g} K_{1}^{(3)} F_{1}$ of $L / K_{1}^{(3)} F_{1}$ is not divisible by 3 , then the 3rank of $\mathrm{Cl}(L)$ is a multiple of 2 by Lemma 5 and then we can conclude $h(L) \neq 3$. In order to show $3 \nmid h\left(K_{g} K_{1}^{(3)} F_{1}\right)$, it suffices to show $3 \|$ $h\left(K_{g} K_{1}^{(3)}\right)$. So we calculate $h\left(K_{g} K_{1}^{(3)}\right)$. For this we calculate the class numbers of the intermediate fields $K_{1}^{(3)} F, K_{1}^{(3)}(\sqrt{-3}), K_{1}^{(3)}(\sqrt{-11})$ of the $V_{4}$-extension $K_{g} K_{1}^{(3)} / K_{1}^{(3)}$. $K_{1}^{(3)} F / F$ is a $D_{3}$-extension and its quadratic subextension is $K F=\mathbf{Q}(\sqrt{-23}, \sqrt{33})$. We have $h(K F)=36$. We calculate the class number of a cubic subextension $F\left(\alpha_{94}\right)$ of $K_{1}^{(3)} F / F$, where $\alpha_{94}^{3}-\alpha_{94}^{2}+6 \alpha_{94}-3=0$. Put $\lambda=\alpha_{94}+\sqrt{-23}$. Then $F\left(\alpha_{94}\right)=\mathbf{Q}(\lambda)$ and $\lambda^{6}-2 \lambda^{5}+82 \lambda^{4}-110 \lambda^{3}+1676 \lambda^{2}-956 \lambda^{2}+7047=0$. The function 'polred' of pari-gp gives a simpler generating polynomial: $\mathbf{Q}(\lambda)=\mathbf{Q}(\theta)$, where $\theta^{6}-3 \theta^{5}+\theta^{4}+3 \theta^{3}-11 \theta^{2}+9 \theta+27=0$. Then KANT gives $h(\mathbf{Q}(\theta))=3$. Hence by Lemma 3 we get $h\left(K_{1}^{(3)} F\right)=12$ and therefore $\mathrm{Cl}\left(K_{1}^{(3)} F\right) \cong C_{12}$. Similarly we get $h\left(K_{1}^{(3)}(\sqrt{-3})\right)=h\left(K_{1}^{(3)}(\sqrt{-11})\right)=4$. Thus, by Lemma 4 we conclude that $h\left(K_{g} K_{1}^{(3)}\right)$ is 3 up to a 2-power. Hence $h(L) \neq 3$.

Next, we show $2 \nmid h(L)$. Assume that $2 \mid h(L)$. Since $\mathrm{Cl}\left(K_{1}^{(3)} F\right) \cong C_{12}$ and $K_{1} / K_{1}^{(3)} F$ is cyclic quartic, by Lemma 9 we have $2 \nmid h\left(K_{1}\right)$. Therefore since $L / K_{1}$ is cyclic cubic, $\mathrm{Cl}(L) \cong V_{4}$ by Lemma 6 . Now we consider the action of $\operatorname{Gal}(L / K) \cong \operatorname{Gal}\left(K F_{1} / K\right) \times C_{12} \cong D_{3} \times C_{12}$ on $\mathrm{Cl}(L)$. This induces a group homomorphism

$$
\rho: D_{3} \times C_{12} \rightarrow \operatorname{Aut}(\mathrm{Cl}(L)) \cong \operatorname{Aut}\left(V_{4}\right) \cong D_{3} .
$$


Obviously 3|| $\operatorname{Ker}(\rho) \mid$ and therefore $\operatorname{Gal}\left(L / K_{1}\right)$ or $\operatorname{Gal}\left(L / K_{1}^{(2)} F\right)$ acts trivially on $\mathrm{Cl}(L)$. Hence $4 \mid h\left(K_{1}\right)$ or $4 \mid h\left(K_{1}^{(2)} F\right)$. We already see $2 \nmid h\left(K_{1}\right)$. Thus we calculate $h\left(K F_{1}\right) . K F_{1} / K$ is a $D_{3}$-extension and $K\left(\alpha_{1}\right)$ is its cubic subextension. As above, we get $h\left(K\left(\alpha_{1}\right)\right)=24$ and $h\left(K F_{1}\right)=12$ by Lemma 3. Since $K_{1}^{(2)} F_{1} / K F_{1}$ is cyclic quartic, $2 \nmid h\left(K_{1}^{(2)} F\right)$ by Lemma 9. This is a contradiction. Hence $h(L)=1$. Thus, $K_{u r}=L$.

$d=-840$. We have $\mathrm{Cl}(K) \cong C_{2}^{3}, K_{1}=\mathbf{Q}(\sqrt{-2}, \sqrt{-3}, \sqrt{5}, \sqrt{-7})$, and $h\left(K_{1}\right)=4$. We will show $K_{u r}=K_{2}$. Since $r d_{K}=\sqrt{840}=28.9827 \ldots$ and $r d_{K}<B(2 \cdot 8 \cdot 4 \cdot 11)([38])$, we have $\left[K_{u r}: K_{2}\right]<16$. We first show that $h\left(K_{2}\right)$ is odd. Since $\mathrm{Cl}(\mathbf{Q}(\sqrt{5}, \sqrt{-42})) \cong C_{4}^{2}$, we have $\mathrm{Cl}\left(K_{1}\right) \cong V_{4}$. Hence by Lemma 9 , the 2-class group of $K_{2}$ is cyclic. Assume that $h\left(K_{2}\right)$ is even. Let $M$ be the unique quadratic subextension of $K_{3} / K_{2}$. Then obviously $M$ is normal over $K$. We put $\Lambda=\operatorname{Gal}(M / K)$. Then $\Lambda$ is a group of order 64 such that

$$
\Lambda / \Lambda^{\prime} \cong C_{2}^{3}, \Lambda^{\prime} / \Lambda^{\prime \prime} \cong V_{4}, \Lambda^{\prime \prime} / \Lambda^{\prime \prime \prime} \cong C_{2} .
$$

However, any group of order 64 has abelian derived group (see [17]). This is a contradiction. Thus, $h\left(K_{2}\right)$ is odd and therefore $\mathrm{Cl}\left(K_{2}\right)$ is trivial or $\mathrm{Cl}\left(K_{2}\right) \cong C_{3}^{2}$ by Proposition 2. (Note that $K_{g}=K_{1}$ in this case.)

Assume $\mathrm{Cl}\left(K_{2}\right) \cong C_{3}^{2}$. Put $\Gamma=\operatorname{Gal}\left(K_{2} / \mathrm{Q}\right)$. The action of $\Gamma$ on $\mathrm{Cl}\left(K_{2}\right)$ induces a group homomorphism

$$
\rho: \Gamma \rightarrow \operatorname{Aut}\left(\mathrm{Cl}\left(K_{2}\right)\right) \cong \operatorname{Aut}\left(C_{3}^{2}\right) \cong \mathrm{GL}(2,3) .
$$

By Lemma 8 the image of $\rho$ is isomorphic to $D_{4}$, because if $|\operatorname{Im}(\rho)| \leqq 4$, then $\rho$ has an abelian image and the same argument as in the proof of Proposition 2 shows that $h\left(K_{1}\right)$ must be divisible by 9 . Let $F$ be the field corresponding to $\operatorname{Ker}(\rho)$. Then $F$ is normal over $\mathbf{Q}$ and $\operatorname{Gal}(F / \mathbf{Q}) \cong D_{4}$. Let $E$ be the quadratic subfield of $F$ such that $F / E$ is cyclic and $E^{\prime}$ the intermediate field of $F / E$. If $E^{\prime} / E$ is ramified, then $F / E$ is totally ramified. Therefore $E^{\prime} / E$ must be unramified because all the inertia groups of primes of $F$ have order at most two. We take the quadratic subfield $\mathbf{Q}\left(\sqrt{d^{\prime}}\right)$ of $K_{1}$ such that the primes ramified in this field coincide with those ramified in $F$. Then $F\left(\sqrt{d^{\prime}}\right) / \mathbf{Q}\left(\sqrt{d^{\prime}}\right)$ is unramified at all finite primes. Thus, if $\mathbf{Q}\left(\sqrt{d^{\prime}}\right)$ is contained in $F$, then $F / E^{\prime}$ is unramified at all finite primes, but this is impossible, because any quadratic subfield of $K_{1}$ has narrow class group of exponent at most two. Hence $F\left(\sqrt{d^{\prime}}\right)$ is a $D_{4} \times C_{2}$-extension of $\mathbf{Q}$. Then $F\left(\sqrt{d^{\prime}}\right) / E^{\prime}$ is a $V_{4}$-extension. Let $F^{\prime}$ be the third quadratic subextension of it. Then $F^{\prime}$ is a $D_{4}$-extension of $\mathrm{Q}$. There exists a prime $p$ that is ramified in $F / Q$ but not ramified in $E^{\prime} / \mathrm{Q}$. Then the prime divisors of $p$ 
in $E^{\prime}$ must be ramified in both $F / E^{\prime}$ and $F^{\prime} / E^{\prime}$. Since $F\left(\sqrt{d^{\prime}}\right) / E^{\prime}\left(\sqrt{d^{\prime}}\right)$ is unramified, these divisors are ramified also in $E^{\prime}\left(\sqrt{d^{\prime}}\right) / E^{\prime}$. Thus, the inertia group of any prime divisor of $p$ in $F\left(\sqrt{d^{\prime}}\right)$ is isomorphic to $V_{4}$. This is a contradiction. Hence $\mathrm{Cl}\left(K_{2}\right)$ is trivial and $K_{u r}=K_{2}$.

Now we determine $G=\operatorname{Gal}\left(K_{2} / K\right)$. $G$ is a group of order 32 such that

$$
G / G^{\prime} \cong C_{2}^{3}, G^{\prime} \cong V_{4}
$$

There are 9 (nonisomorphic) such groups, which belong to the family $\Gamma_{4}$ (see [17]). Among them, the groups having an abelian subgroup isomorphic to $C_{4}^{2}$ (note that $\left.\operatorname{Gal}\left(K_{2} / \mathrm{Q}(\sqrt{5}, \sqrt{-42})\right) \cong C_{4}^{2}\right)$ are $32 \Gamma_{4} a_{2}, 32 \Gamma_{4} a_{3}, 32 \Gamma_{4} c_{2}$, $32 \Gamma_{4} c_{3}$, and $32 \Gamma_{4} d$. Let $N$ be any quadratic subextension of $K_{1} / K$ other than $\mathbf{Q}(\sqrt{5}, \sqrt{-42})$, that is, any of the fields $\mathbf{Q}(\sqrt{-2}, \sqrt{105}), \mathbf{Q}(\sqrt{-3}, \sqrt{70})$, $\mathbf{Q}(\sqrt{-7}, \sqrt{30}), \mathbf{Q}(\sqrt{6}, \sqrt{-35}), \mathbf{Q}(\sqrt{-10}, \sqrt{21})$, and $\mathbf{Q}(\sqrt{14}, \sqrt{-15})$, and put $H=\operatorname{Gal}\left(K_{2} / N\right)$. We have $\mathrm{Cl}(N) \cong C_{4} \times C_{2}$. Hence $H$ is a group of order 16 such that

$$
H / H^{\prime} \cong C_{4} \times C_{2}, H^{\prime} \cong C_{2} .
$$

Therefore $H$ is isomorphic to $16 \Gamma_{2} c_{1}, 16 \Gamma_{2} c_{2}$, or $16 \Gamma_{2} d$. Thus, $G$ is isomorphic to $32 \Gamma_{4} c_{3}$, or $32 \Gamma_{4} d$, according as $G$ has a quotient group isomorphic to $16 \Gamma_{2} a_{2}\left(\cong Q_{8} \times C_{2}\right)$, or not. (All these arguments work also for $K=$ $\mathbf{Q}(\sqrt{-420})$. We have $h\left(\mathbf{Q}(\sqrt{-420})_{1}\right)=4$ and $\operatorname{Gal}\left(K_{2} / \mathbf{Q}(\sqrt{5}, \sqrt{-21})\right) \cong$ $C_{4}^{2}$.) If $K$ has an unramified $Q_{8}$-extension, then the composite field of it with $K_{1}$ is a $Q_{8} \times C_{2}$-extension of $K$. Since $32 \Gamma_{4} c_{3}$ does not have two normal subgroups with quotient isomorphic to $Q_{8} \times C_{2}$, we need only to judge whether $K$ has an unramified $Q_{8}$-extension which is normal over $\mathbf{Q}$. F. Lemmermeyer gives a criterion for this [30]. By his result $K$ does not have such an extension. Hence $G \cong 32 \Gamma_{4} d$. (Since $\mathrm{Q}(\sqrt{-420})$ has an unramified $Q_{8}$-extension which is normal over $\mathbf{Q}, \operatorname{Gal}\left(\mathbf{Q}(\sqrt{-420})_{u r} / \mathbf{Q}(\sqrt{-420})\right) \cong$ $32 \Gamma_{4} c_{3}$.)

$d=-920$. We have $\mathrm{Cl}(K) \cong C_{10} \times C_{2}, K_{g}=\mathrm{Q}(\sqrt{2}, \sqrt{5}, \sqrt{-23})$, and $h\left(K_{g}\right)=60$. Put $L=\left(K_{g}\right)_{1}$. We will show $K_{u r}=L$. Since $r d_{K}=$ $\sqrt{920}=30.3315 \ldots$ and $r d_{K}<B(2 \cdot 4 \cdot 60 \cdot 4)([38])$, we have $\left[K_{u r}: L\right]<4$. Since $\mathrm{Cl}^{(2)}(K) \cong V_{4}$, the 2-class field tower of $K$ terminates with $K_{2}^{(2)}$ and therefore $2 \nmid h\left(K_{2}^{(2)}\right)$. Since $K_{1} K_{2}^{(2)} / K_{2}^{(2)}$ is cyclic quintic, $2 \nmid h\left(K_{1} K_{2}^{(2)}\right)$ by Lemma 5 and since $L / K_{1} K_{2}^{(2)}$ is cyclic cubic, $2 \nmid h(L)$ by Lemma 5 again.

Next, we show $3 \nmid h(L)$. Put $F=\mathbf{Q}(\sqrt{5}, \sqrt{-46})$. This is one of the intermediate fields of $K_{g} / K$. We have $\mathrm{Cl}(F) \cong C_{40}$. Hence $K_{2}^{(2)} / F$ is 
cyclic octic and therefore also $K_{2}^{(2)}\left(\alpha_{1}\right) / F\left(\alpha_{1}\right)$ is cyclic octic. Note that $h(\mathbf{Q}(\sqrt{-23}))=3$ and $\mathbf{Q}(\sqrt{-23})_{1}=\mathbf{Q}\left(\sqrt{-23}, \alpha_{1}\right)$, where $\alpha_{1}^{3}-\alpha_{1}-1=0$ and that $\left(K_{g}\right)_{1}^{(3)}=K_{g}\left(\alpha_{1}\right)$. Therefore $3 \nmid h\left(\left(K_{g}\right)_{1}^{(3)}\right)$ by Lemma 9 and then $3 \nmid h\left(K_{2}^{(2)}\left(\alpha_{1}\right)\right)$ by Lemma 5 . Since $L / K_{2}^{(2)}\left(\alpha_{1}\right)$ is cyclic quintic, $3 \nmid h(L)$ by Lemma 5 . Hence $h(L)=1$ and therefore $K_{u r}=L=K_{2}$.

We easily check that $\operatorname{Gal}\left(K_{u r} / K\right) \cong \operatorname{Gal}\left(K_{2}^{(2)}\left(\alpha_{1}\right) / K\right) \times \operatorname{Gal}\left(K_{1}^{(5)} / K\right)$. By the result of $\mathrm{H}$. Kisilevsky [25], we have $\operatorname{Gal}\left(K_{2}^{(2)} / K\right) \cong Q_{16}$. Therefore $\operatorname{Gal}\left(K_{u r} / K\right) \cong\left(Q_{16} \ltimes C_{3}\right) \times C_{5}$.

Finally, we note that the maximal unramified extension of $Q(\sqrt{-920})$ has degree 480 and is the field with class number one of the largest degree we know (under GRH). (J. Martinet gave an example of a field with class number one and degree 116 [33]. (This is an unconditional result.))

\section{QUADRATIC NUMBER FIELDS HAVING AN UNRAMIFIED EXTENSION NOT CONTAINED IN $\left(K_{g}\right)_{1}$}

We explain here that from some quartic number fields of type $S_{4}$ we can obtain quadratic number fields whose genus fields have an unramified $A_{4}$-extension.

First, we review the following general fact.

Proposition 6. ([26]. See also [56].) Let $E$ be an algebraic number field of degree $n \geqq 3$. If the discriminant $d_{E}$ of $E$ is a fundamental quadratic discriminant, that is, a discriminant of a quadratic number field, then the normal closure $M$ of $E$ is an $S_{n}$-extension of $\mathbf{Q}$ and is unramified at all finite primes over its quadratic subfield $\mathbf{Q}\left(\sqrt{d_{E}}\right)$.

From this, any number field $E$ of degree $n \geqq 3$ whose discriminant is fundamental quadratic yields a quadratic number field $\mathbf{Q}\left(\sqrt{d_{E}}\right)$ having an unramified $A_{n}$-extension. (If $d_{E}>0$ and $E$ is not totally real, then the infinite primes are ramified.) Moreover, $E$ yields infinitely many quadratic number fields having an unramified $S_{n}$-extension. In fact, for any quadratic number field $K \neq \mathbf{Q}\left(\sqrt{d_{E}}\right)$ with $d_{K}=d_{E} d^{\prime}$, where $d^{\prime}$ is a fundamental quadratic discriminant, the composite field $K M$ is an unramified $S_{n}$-extension of $K$. (The unramifiedness of $K \mathrm{Q}\left(\sqrt{d_{E}}\right) / K$ follows by genus theory.) In this case, since $K M$ and $K_{g}$ are linearly disjoint over $K \mathrm{Q}\left(\sqrt{d_{E}}\right), K_{g}$ has an unramified $A_{n}$-extension. (This is the case also when $K=\mathrm{Q}\left(\sqrt{d_{E}}\right)$.) Therefore, if $n \geqq 4$, then $K_{g} M$ is an unramified extension not contained in $\left(K_{g}\right)_{1}$. In particular, if $n=4$, then the (narrow) class field tower of the 
field $\mathbf{Q}\left(\sqrt{d_{E}}\right)$ has length at least two and the (narrow) class field tower of $K$ has length at least three if $d \neq d_{E}$.

Now we describe the field $K=\mathrm{Q}(\sqrt{-687})$. The factorization of -687 is $-687=(-3) \cdot 229$ and 229 is the first prime discriminant of totally imaginary quartic number fields. Let $E$ be a quartic number field with discriminant 229 (there exists only one such field up to isomorphism [14]) and $M$ its normal closure. Then by the proposition above, $M$ is an $S_{4}$-extension of $\mathbf{Q}$ which is an $A_{4}$-extension of the real quadratic number field $F:=\mathrm{Q}(\sqrt{229})$ unramified at all finite primes (abbrev. weakly-unramified). We can show that the class number of $M$ is one by the method described in $\S 8$ plus some additional consideration. Since by the table in [12], $B(24 \cdot 60,0,12 \cdot 60)>$ $r d_{F}=\sqrt{229}, M$ has no nontrivial weakly-unramified extension. (This is unconditional.) Hence $M$ is the maximal weakly-unramified extension of $F$. (While $F_{u r}=F_{1},\left[F_{1}: F\right]=3$.) Since $\mathrm{Cl}(K) \cong C_{12}$, the composite field $K_{1} M$ has degree 288. By the table in [38], $B(576,0,288)>r d_{K}=\sqrt{687}$ under GRH. Therefore $K_{u r}=K_{1} M$ under GRH. Now we determine the structure of the Galois group $G=\operatorname{Gal}\left(K_{u r} / K\right)$. Obviously $K_{1}^{(2)} M$ and $K_{1}^{(3)}$ are linearly disjoint over $K$ and therefore $G$ is isomorphic to the direct product $H=\operatorname{Gal}\left(K_{1}^{(2)} M / K\right)$ with $\operatorname{Gal}\left(K_{1}^{(3)} / K\right) \cong C_{3}$. We consider the structure of $H$. Let $C$ be a cubic subextension of $K M / K$. Then $C$ corresponds to a Sylow 2-subgroup of $H$ and $\operatorname{Gal}(K M / E)$ is isomorphic to a Sylow 2-subgroup of $\operatorname{Gal}(K M / K) \cong S_{4}$, that is, isomorphic to $D_{4}$. Thus, if we put $T=\operatorname{Gal}\left(K_{1}^{(2)} M / C\right)$, then we have

$$
T / T^{\prime} \cong C_{4} \times C_{2}, T^{\prime} \cong C_{2} .
$$

Since $\operatorname{Gal}\left(K_{1}^{(2)} M / K_{g} F_{1}\right) \cong C_{2}^{3}$, we have $T \cong 16 \Gamma_{2} c_{1} \cong D_{4} \curlywedge C_{4}$. Hence $T$ contains a cyclic subgroup $W$ of order four containing $\operatorname{Gal}\left(K_{1}^{(2)} M / K M\right)$ with $T \cap W=\{1\}$, and this group and $\operatorname{Gal}\left(K_{1}^{(2)} M / K_{1}^{(2)}\right)$ generate $H$. Therefore $H \cong A_{4} \rtimes C_{4}$. (We note that $H$ is a nonsplit extension of $\operatorname{Gal}(K M / K) \cong S_{4}$ by $C_{2}$. But $H$ is not a double cover of $S_{4}$.) Thus, $G \cong\left(A_{4} \rtimes C_{4}\right) \times C_{3}$.

Some quartic number fields whose discriminants are fundamental quadratic yield infinitely many quadratic number fields with class field tower of length at least four.

Proposition 7. (See [45]) Let $E$ be a quartic number field with $d_{E}=-p$ $(p \equiv 3(\bmod 4)$ a prime). Then $E$ is embedded into an octic number field $\widetilde{E}$ whose normal closure is an $\widetilde{S_{4}}$-extension of $\mathbf{Q}$ and is unramified over its quadratic subfield $\mathbf{Q}(\sqrt{-p})$, where $\widetilde{S_{4}}$ is the double cover of $S_{4}$ which is 
characterized by the property that a transposition in $S_{4}$ lifts to an element of order two, while a product of two disjoint transpositions lifts to an element of order four: $\widetilde{S_{4}} \cong \mathrm{GL}(2,3)$.

From this, from a quartic number field $E$ with $d_{E}=-p \equiv 1(\bmod 4)$, we get an unramified $\widetilde{A}_{4}$-extension of the imaginary quadratic number field $\mathbf{Q}(\sqrt{-p})$. We note that from the data for quartic number fields of signature $(2,1)[13]$, the quartic prime discriminants $-p \equiv 1(\bmod 4)$ with $p \leqq 2003$ are $-283,-331,-491,-563,-643,-751,-1399,-1423,-1823,-1879$, and -1931 . All such fields give two dimensional complex projective linear representation

$$
\rho: \operatorname{Gal}(\overline{\mathbf{Q}} / \mathbf{Q}) \rightarrow \operatorname{PGL}(2, \mathbf{C})
$$

of the absolute Galois group $\operatorname{Gal}(\overline{\mathbf{Q}} / \mathbf{Q})$ of $\mathbf{Q}$ of type $S_{4}$ which has a lifting

$$
\tilde{\rho}: \operatorname{Gal}(\overline{\mathbf{Q}} / \mathbf{Q}) \rightarrow \mathrm{GL}(2, \mathbf{C})
$$

with conductor $p$ and odd determinant (see [45, §8]). (A. Jehanne [23] has studied the general embedding problem $\widetilde{S_{4}} \rightarrow S_{4}$, especially for the case where the base field is $\mathbf{Q}$. We find in [23] a generating polynomial of $\widetilde{E}$ for $p=283,331,491,563,643,751$. Field $E$ in Proposition 7 yields infinitely many quadratic number fields with class field tower of length at least four. Let $\widetilde{M}$ be the normal closure of $\widetilde{E}$. Then $\widetilde{M}$ is an $\widetilde{S_{4}}$-extension of $\mathbf{Q}$. For any quadratic number field $K$ such that $d_{K} / d_{E}$ is fundamental quadratic, the composite field $K \widetilde{M}$ is an unramified $\widetilde{S_{4}}$-extension of $K$. Then $K$ has (narrow) class field tower of length at least four. For example, let $K=$ $\mathrm{Q}(\sqrt{-5 \cdot 283})$. Since $\mathrm{Cl}(K) \cong C_{34} \cong C_{2} \times C_{17}, \operatorname{Gal}\left(K_{1} \mathbf{Q}(\sqrt{-283})_{u r} / K\right) \cong$ $\widetilde{S_{4}} \times C_{17}$ and therefore $\left[K_{1} \mathrm{Q}(\sqrt{-283})_{u r}: \mathrm{Q}\right]=1632$. Even under GRH, we get only $\left[K_{u r}: K_{1} \mathrm{Q}(\sqrt{-283})_{u r}\right]<44$ from Odlyzko's bound, however, it is thought likely that $K_{u r}=K_{1} \mathbf{Q}(\sqrt{-283})_{u r}$ (this implies $K_{u r}=K_{4}=$ $K_{1} \mathbf{Q}(\sqrt{-283})_{u r}$ ), but no proof has yet been obtained. (We can prove $K_{3}=$ $K_{1} \mathbf{Q}(\sqrt{-283})_{2}$.)

There exist many quartic number fields of type $S_{4}$ whose discriminants are not fundamental quadratic which yield infinitely many quadratic number fields having an unramified $S_{4}$-extension.

We will not consider here general such fields. We decribe only such quartic number fields giving unramified $S_{4}$-extensions of $\mathrm{Q}(\sqrt{-856})$ and $\mathrm{Q}(\sqrt{-996})$.

Let $E$ be a quartic number field defined by the (irreducible) polynomial $f(X)=X^{4}-2 X^{3}+5 X^{2}-2 X-1$ and $M$ its normal closure. We easily 
see $\operatorname{Gal}(M / \mathrm{Q}) \cong S_{4}$ and $d_{E}=-6848=-2^{6} \cdot 107$. Let $K=\mathrm{Q}(\sqrt{-856})$. Note that $-856=8 \cdot(-107)$. We see that $K M / K$ is an unramified $S_{4^{-}}$ extension. For this, it suffices to show that $K E / K$ is unramified. We can easily get a generating polynomial of $K E$. For example, we can easily calculate the minimal polynomial of $\theta+\sqrt{-214}$, where $\theta$ is a root of $f(X)$ with $E=\mathbf{Q}(\theta)$. From this we get as a generating polynomial of $K E$, $X^{8}-2 X^{7}+15 X^{6}+20 X^{5}+25 X^{4}+228 X^{3}+573 X^{2}+170 X+25$ by using the function 'polred' of pari-gp. Then we get $d_{K E}=2^{12} \cdot 107^{4}=d_{E}^{4}$ by using 'discf' of pari-gp. Hence $K M / K$ is unramified. For any quadratic number field $F$ such that $856 \mid d_{F}$ and that the localization of $F$ at the prime divisor of 2 in $F$ is (isomorphic to) the one of $K$ at the prime divisor of 2 in $K, F M / F$ is an $S_{4}$-extension unramified at all finite primes.

Let $E^{\prime}$ be a quartic number field defined by the (irreducible) polynomial $X^{4}-3 X^{2}-2 X+1$ and $M^{\prime}$ its normal closure. We easily see $\operatorname{Gal}\left(M^{\prime} / \mathrm{Q}\right) \cong$ $S_{4}$ and $d_{E^{\prime}}=-1328=-2^{4} \cdot 83$. Let $K^{\prime}=\mathrm{Q}(\sqrt{-996})$. Note that $-996=$ $(-4) \cdot(-3) \cdot(-83)$. As above we can show that $K^{\prime} M^{\prime} / K^{\prime}$ is an unramified $S_{4}$-extension.

We note that the discriminants of the fields $E$ and $E^{\prime}$ above are of the form $f^{2} d^{\prime}$, where $d^{\prime}$ is a fundamental discriminant with $h\left(\mathbf{Q}\left(\sqrt{d^{\prime}}\right)\right)=3$ and $f=8=d_{\mathbf{Q}(\sqrt{2})}$, or $f=-4=d_{\mathbf{Q}(\sqrt{-1})}$. We also note that the ramification of the prime 2 in $M / \mathrm{Q}$ (resp. $M^{\prime} / \mathrm{Q}$ ) occurs over the subfield of $M$ (resp. $M^{\prime}$ ) which is an $S_{3}$-extension of $\mathbf{Q}$.

\section{UNRAMIFIED NONSOLVABLE GALOIS EXTENSIONS OF IMAGINARY QUADRATIC NUMBER FIELDS}

For imaginary quadratic number fields $K$ of small conductors we considered, the maximal unramified extension $K_{u r}$ is the top of the class field tower of $K$. Then the following natural question arises: What is the first imaginary quadratic number field having an unramified nonsolvable Galois extension? Recent data for quintic number fields enable us to give a partial answer:

Proposition 8. The field $\mathbf{Q}(\sqrt{-1507})$ is the first imaginary quadratic number field having an unramified $A_{5}$-extension which is normal over $\mathbf{Q}$ in the sense that none of $\mathbf{Q}(\sqrt{d})$ of discriminant $d$ with $0>d>-1507$ has such an extension. Moreover, such an extension of $K=\mathbf{Q}(\sqrt{-1507})$ is given by the composite field of $K$ with the splitting field of the quintic polynomial $X^{5}-5 X^{3}+5 X^{2}+24 X+4$, which is an $A_{5}$-extension of $\mathbf{Q}$.

Proof. From the table in [2], the splitting field of the quintic polynomial 
$X^{5}-5 X^{3}+5 X^{2}+24 X+4$ is an $A_{5}$-extension of $\mathrm{Q}$ and the only two primes 11 and 137 are ramified in this field with ramification index two. Therefore the composite field of $\mathbf{Q}(\sqrt{-1507})$ with it is an unramified $A_{5}$ extension of $\mathbf{Q}(\sqrt{-1507})$ (note that the factorization of 1507 is $1507=$ $11 \cdot 137)$, which is an $A_{5} \times C_{2}$-extension of $\mathbf{Q}$. Now we show that none of $\mathbf{Q}(\sqrt{d})$ of discriminant $d$ with $0>d>-1507$ has such an extension. Let $L$ be an unramified $A_{5}$-extension of a quadratic number field $F$ which is normal over $\mathbf{Q}$. Then the Galois group $\mathrm{Gal}(L / \mathrm{Q})$ is isomorphic to $S_{5}$ or $A_{5} \times C_{2}$. If $\operatorname{Gal}(L / \mathrm{Q}) \cong S_{5}$, then $L$ is the normal closure of its quintic subfield $E$ and the discriminant $d_{E}$ of $E$ coincides with the one $d_{F}$ of $F$ (see [26]). Since the maximal discriminant of quintic number fields of signature $(3,1)$ (whose normal closures are $S_{5}$-extensions of $\mathrm{Q}$ ) is -4511 and the minimal discriminant of quintic number fields of signature $(1,2)$ is 1609 ([44]), $\mathbf{Q}(\sqrt{-4511})$ is the first imaginary quadratic number field having an unramified $A_{5}$-extension which is an $S_{5}$-extension of $\mathrm{Q}$. Now, we assume $\operatorname{Gal}(L / \mathrm{Q}) \cong A_{5} \times C_{2}$ and let $M$ be the unique subfield of $L$ which is an $A_{5}$-extension of $\mathrm{Q}$. Since $L=M F / F$ is unramified, the primes ramified in $M$ are prime divisors of $d_{F}$ and their ramification indices are all two. Hence if we let $E$ be a quintic subfield of $M$, we get $d_{F}^{2} \mid d_{E}$. From the data for quintic number fields in [44] and the table in [3], $\mathrm{Q}(\sqrt{-1507})$ is the first imaginary quadratic number field having an unramified $A_{5}$-extension which is an $A_{5} \times C_{2}$-extension of $\mathbf{Q}$.

Remark. Probably, the condition "which is normal over Q" is unnecessary. It is reasonable to expect this. In fact, if we assume that GRH is true, Odlyzko's discriminant bounds enable us to show that most of $\mathbf{Q}(\sqrt{d})$ with $0>d>-1507$ do not have an unramified $A_{5} \times A_{5}$-extension. (We can show that under GRH, except for $d=-1387,-1451,-1459,-1480,-1483,-1492$, and -1499 , none of $\mathbf{Q}(\sqrt{d})$ with $0>d>-1507$ has any unramified $A_{5}$ extension.) We expect that in future data for number fields of degree ten will enable us to eliminate the condition above; If an $A_{5}$-extension $L$ of a quadratic number field $K$ is not normal over $\mathbf{Q}$, its normal closure $\widetilde{L}$ has Galois group isomorphic to $A_{5} \times A_{5}$ over $K$. Let $E$ be a quintic subextension of $L / K$. Then $E$ has degree ten. The five conjugate fields of $E$ which are conjugate over $K$ generate $L$ and the other five conjugate fields generate another $A_{5}$-extension of $K$. Thus $\operatorname{Gal}(\tilde{L} / K) \cong A_{5} \times A_{5}$.

We expect stronger assertion that $\mathbf{Q}(\sqrt{-1507})$ is the first imaginary quadratic number field having an unramified nonsolvable Galois extension. We exhibit here two other examples of an imaginary quadratic number field having an unramified nonsolvable Galois extension. $Q(\sqrt{-14731})$ has an unramified $A_{6}$-extension which is an $S_{6}$-extension of $\mathbf{Q}$. Such an extension 
is given as the splitting field of the sextic polynomial $X^{6}+3 X^{5}+5 X^{4}+$ $4 X^{3}+3 X^{2}+2 X+1$. $\mathbf{Q}(\sqrt{-30759})$ has an unramified PSL $(2,7)$-extension which is a $\operatorname{PSL}(2,7) \times C_{2}$-extension of $\mathbf{Q}$. Such an extension is given as the composite field of $Q(\sqrt{-30759})$ with the splitting field of the septic polynomial $X^{7}+2 X^{6}-3 X^{4}-X^{3}-X^{2}-X+2$. The fact that this splitting field is a PSL $(2,7)$-extension of $\mathbf{Q}$ was found by $\mathrm{K}$. Yamazaki [61].

As explained in $\S 2$, under GRH, if $|d| \leqq 2003$, then $\left[K_{u r}: K\right]<\infty$ for $K=\mathrm{Q}(\sqrt{d}), d=d_{K}<0$. In this range, we only know one other field having an unramified nonsolvable Galois extension, namely, the field with $d=-1959=(-3) \cdot 653$. This is the second imaginary quadratic number field having an unramified $A_{5}$-extension which is normal over $\mathrm{Q}$. Let $L$ be the splitting field of the quitic polynomial $X^{5}+X^{3}+62 X^{2}+104 X+169$. Then $L$ is an imaginary $A_{5}$-extension of $\mathbf{Q}$, in which (except for the infinite prime) only the finite prime 653 is ramified and its ramification index is two ([3]). Thus, for any quadratic number field $K$ whose discriminant is a multiple of 653 , the compositum $K L$ is an $A_{5}$-extension of $K$ unramified at all finite primes. (If $K$ is real, the infinite primes are ramified in $K L / K$.) We note that $F=\mathrm{Q}(\sqrt{653})$ is the first real quadratic number field having an weakly-unramified $A_{5}$-extension which is normal over $\mathrm{Q} . F$ has narrow class number one. Therefore $F$ has no nontrivial weakly-unramified abelian extension. Under GRH from Odlyzko's bound we get $\left[F_{w-u r}: F L\right] \leqq 3$ and therefore $\operatorname{Gal}\left(F_{w-u r} / F\right)$ is isomorphic to $A_{5}$, or a nonsplit extension of $A_{5}$ by $C_{2}$ or $C_{3}$.

The third imaginary quadratic number field having an unramified $A_{5^{-}}$ extension which is normal over $\mathbf{Q}$ is $\mathbf{Q}(\sqrt{-2083})$. Moreover, the $A_{5}$ extension of $K=\mathrm{Q}(\sqrt{-2083})$ is given by the composite field of $K$ with the splitting field of the quintic polynomial $X^{5}+8 X^{3}+7 X^{2}+172 X+53$, which is an $A_{5}$-extension of $\mathbf{Q}$.

Now we return to the field $\mathrm{Q}(\sqrt{-1507})$. We have $\mathrm{Cl}(K) \cong C_{4}$. The Hilbert class field $K_{1}$ of $K$ is $K(\sqrt{(-23+3 \sqrt{137}) / 2})$ and its class number is one. Thus, $K_{1}$ is an imaginary $D_{4}$-extension of $\mathbf{Q}$ with class number one having an unramified $A_{5}$-extension. This field is only example we know of an imaginary normal extension of $\mathbf{Q}$ with class number one having an unramified nonsolvable Galois extension. (On the other hand, there exist many (probably infinitely many) real quadratic number fields with class number one having an unramified $A_{n}$-extension for each $n \geqq 5[55,60]$.) 


\section{ApPENDix 1. Calculation of Class NUMBERS OF $S_{4}$-EXTENSIONS OF $\mathbf{Q}$}

Let $M$ be an $S_{4}$-extension of $\mathbf{Q}$. We consider here how to calculate its class number. If we know the class numbers of a cubic subfield and sextic subfields containing it, we get the class number $h(M)$ of $M$ up to a power of two by using class number relation for $V_{4}$-extension repeatedly. Let $L$ be the unique normal subfield of $M$ which is an $S_{3}$-extension of Q. Then $M / L$ is a $V_{4}$-extension and three intermediate fields are conjugate. Therefore, if we know the class numbers of $L$ and any one of the intermediate fields of $M / L$, then we get $h(M)$ up to a 2-power. Let $E$ be a cubic subfield of $L$. Then $M / E$ is a $D_{4}$-extension and the $V_{4}$-subextension $N$ of $M / E$ is an intermediate field of $M / L$. Thus, if the class numbers of three intermediate fields of $N / E$, which are sextic, are known, we get $h(N)$ up to a 2-power.

Now, we give the sextic fields more explicitly by starting with a quartic subfield of $M$. Let $F$ be a quartic subfield of $M$ and $f(X)=X^{4}+a_{1} X^{3}+$ $a_{2} X^{2}+a_{3} X+a_{4} \in \mathbf{Z}[X]$ its generating polynomial, that is, $F=\mathbf{Q}(\beta)$, where $\beta$ is a root of $f(X)$. Then the resolvent cubic $f_{r}(X)=X^{3}-a_{2} X^{2}+$ $\left(a_{1} a_{3}-4 a_{4}\right) X-a_{4}\left(a_{1}^{2}-4 a_{2}\right)-a_{3}^{2}$ of $f(X)$ is irreducible over $\mathbf{Q}$ and its roots are $\alpha=\beta_{1} \beta_{2}+\beta_{3} \beta_{4}, \beta_{1} \beta_{3}+\beta_{2} \beta_{4} \beta_{1} \beta_{4}+\beta_{2} \beta_{3}$, where $\beta_{1}, \beta_{2}, \beta_{3}$, and $\beta_{4}$ are the roots of $f(X)$. Let $E=\mathbf{Q}(\alpha)$ and $N$ the $V_{4}$-subextension of $M / E$. We want to know the three intermediate fields of $N / E$ and their class numbers. One is $L=K E=E(\sqrt{d})$, which is the splitting field of $f_{r}(X)$, where $K$ is the unique quadratic subfield of $M$ and $d$ its discriminant. Other one is $\mathbf{Q}\left(\beta_{1} \beta_{2}\right)=\mathbf{Q}\left(\beta_{1} \beta_{2}-\beta_{3} \beta_{4}\right)$. Note that $\left(\beta_{1} \beta_{2}-\beta_{3} \beta_{4}\right)^{2}=\left(\beta_{1} \beta_{2}+\beta_{3} \beta_{4}\right)^{2}-$ $4 \beta_{1} \beta_{2} \beta_{3} \beta_{4}=\alpha^{2}-4 a_{4}$ and we can write this field as $E\left(\sqrt{\alpha^{2}-4 a_{4}}\right)$. Thus, the other one is $E\left(\sqrt{d\left(\alpha^{2}-4 a_{4}\right)}\right)=\mathbf{Q}\left(\sqrt{d\left(\alpha^{2}-4 a_{4}\right)}\right)=\mathbf{Q}\left(\sqrt{d}\left(\beta_{1} \beta_{2}-\right.\right.$ $\left.\beta_{3} \beta_{4}\right)$ ). The minimal polynomial $g(X)$ of $\beta_{1} \beta_{2}$ is

$$
\begin{aligned}
X^{6}-a_{2} X^{5}+\left(a_{1} a_{3}-a_{4}\right) X^{4}+\left(-a_{1}^{2} a_{4}+2 a_{2} a_{4}-a_{3}^{2}\right) X^{3}+ \\
\left(a_{1} a_{3} a_{4}-a_{4}^{2}\right) X^{2}-a_{2} a_{4}^{2} X+a_{4}^{3}
\end{aligned}
$$

and its discriminant is $d_{f}^{2} a_{4}^{6}\left(-2 a_{1}^{2} a_{3}^{2} a_{4}+a_{1}^{4} a_{4}^{2}+a_{3}^{4}\right)$, where $d_{f}$ is the discriminant of $f(X)$. The minimal polynomial $u(X)$ of $\sqrt{d}\left(\beta_{1} \beta_{2}-\beta_{3} \beta_{4}\right)$ is

$$
\begin{aligned}
& X^{6}-\left(a_{2}^{2}-2 a_{1} a_{3}-4 a_{4}\right) d X^{4}+ \\
& \left(-2 a_{1}^{2} a_{2} a_{4}+a_{1}^{2} a_{3}^{2}+8 a_{1} a_{3} a_{4}-2 a_{2} a_{3}^{2}\right) d^{2} X^{2}-\left(-2 a_{1}^{2} a_{3}^{2} a_{4}+a_{1}^{2} a_{4}^{2}+a_{3}^{4}\right) d^{3}
\end{aligned}
$$

and its discriminant is $64 d_{f}^{11}\left(a_{1} a_{2} a_{3}-a_{1}^{2} a_{4}^{2}-a_{3}^{2}\right)\left(-2 a_{1}^{2} a_{3}^{2} a_{4}+a_{1}^{4} a_{4}^{2}+a_{3}^{4}\right)$. 
We describe ramification. H. J. Godwin [15] proved that the discriminant of $\mathbf{Q}\left(\beta_{1} \beta_{2}\right)$ equals $d_{E} d_{F}$ (see also [24]). In order that the extension $M / K$ is unramified at all finite primes, $d_{F}=d$ is necessary and sufficient (see [26]). In that case, we have also $d_{E}=d$ and therefore $d_{\mathbf{Q}\left(\beta_{1} \beta_{2}\right)}=d^{2}=d_{E}^{2}$. This implies that both $N / \mathbf{Q}\left(\sqrt{d\left(\alpha^{2}-4 a_{4}\right)}\right)$ and $\mathbf{Q}\left(\beta_{1} \beta_{2}\right) / E$ are unramified at all finite primes.

Now we explain how we get $h\left(K_{2}\right)$ for $K=\mathbf{Q}(\sqrt{-643})$. It is a classical result that $K_{2}$ for $K=\mathbf{Q}(\sqrt{-643})$ is an $S_{4}$-extension of $\mathbf{Q}$. So, put $M=K_{2}$ and we use the notations above. Then we can take $f(X)=X^{4}+X^{3}+2 X+1$ and $d_{f}=d=-643$. (From the irreducibility and the squarefreeness of $d_{f}$, we can deduce the fact that the splitting field of $f(X)$ is an $S_{4}$-extension of $\mathbf{Q}$ and is unramified over the unique quadratic subfield $K=\mathbf{Q}(\sqrt{-643})$. (See [56].)) Then $f_{r}(X)=X^{3}-2 X-5$ and we can check $h(E)=2$ by computer. Since $h(K)=3$, by using the class number relation in Lemma 3 , we get $h(L)=4$. (More precisely, by Lemma $5, \mathrm{Cl}(L) \cong V_{4}$.) Now $g(X)=X^{6}+X^{4}-5 X^{3}+X^{2}+1, u(X)=X^{6}-8 \cdot 643 X^{4}+20 \cdot 643^{2} X^{2}+9 \cdot 643^{3}$ and KANT gives $h\left(\mathbf{Q}\left(\beta_{1} \beta_{2}\right)\right)=1$. Hence in this case, the field $\mathbf{Q}\left(\beta_{1} \beta_{2}\right)$ is the Hilbert class field of $E$ and $N / \mathrm{Q}\left(\sqrt{d\left(\alpha^{2}-4 a_{4}\right)}\right)$ is unramified. The function 'polred' of pari-gp reduces $u(X)$ to $X^{6}-3 X^{5}+11 X^{4}-9 X^{3}+$ $23 X^{2}+8 X+57$ and KANT gives $\mathrm{Cl}\left(\mathrm{Q}\left(\sqrt{d\left(\alpha^{2}-4 a_{4}\right)}\right)\right) \cong C_{8}$. We also note that the field $\mathbf{Q}\left(\sqrt{d\left(\alpha^{2}-4 a_{4}\right)}\right)$ is totally imaginary and therefore the narrow class group of it concides with its class group. Then the class number relation for $V_{4}$-extension shows that $h(N)$ is a power of 2 , and since $N$ is the unramified quadratic extension of $\mathbf{Q}\left(\sqrt{d\left(\alpha^{2}-4 a_{4}\right)}\right)$, we get $\mathrm{Cl}(N) \cong C_{4}$. Using the class number relation for $V_{4}$-extension again, $h(M)$ is a power of 2 , and since $M$ is the unramified quartic extension of $\mathbf{Q}\left(\sqrt{d\left(\alpha^{2}-4 a_{4}\right)}\right)$, we get $h(M)=2$. By the same method, we get $h\left(K_{2}\right)=2$ also for $K=\mathbf{Q}(\sqrt{-283})$ and $\mathbf{Q}(\sqrt{-331})$. Moreover, if we take $M$ as the normal closure of the third (resp. fourth, and eighth) quartic number field of type $S_{4}$, which is an unramified $A_{4}$-extension of $\mathrm{Q}(\sqrt{-491})$ (resp. $\mathbf{Q}(\sqrt{-563})$, and $\mathbf{Q}(\sqrt{-751}))$, we get $h(M)=6($ resp. $h(M)=6$, and $h(M)=10)$.

APPENDIX 2. IMAGINARY QUADRATIC NUMBER FIELDS $K$ WITH $K_{2}=\left(K_{g}\right)_{1} \neq K_{1}$

We give here a note on the following.

Problem 1. Characterize the imaginary quadratic number fields $K$ with

$$
K_{2}=\left(K_{g}\right)_{1} \neq K_{1} \text {. }
$$


Let $K$ be an imaginary quadratic number field. The inequality $\left(K_{g}\right)_{1} \neq$ $K_{1}$ is equivalent to $h\left(K_{g}\right)>h(K) /\left[K_{g}: K\right]$ and we can easily calculate $h(K),\left[K_{g}: K\right]$, and $h\left(K_{g}\right)$. Since $K_{g}=K$ if and only if $h(K)$ is odd, for $\left(K_{g}\right)_{1} \neq K_{1}, h(K)$ must be even and therefore the discriminant $d$ of $K$ must have two disctinct prime factors.

On the other hand, it is hard to calculate $h\left(K_{1}\right)$ in general. However, by considering the $p$-class field tower, we can get some necessary condition for $K_{2}=\left(K_{g}\right)_{1}$. Let $p$ be an odd prime. If the class number $h\left(K_{1}^{(p)}\right)$ of the Hilbert $p$-class field $K_{1}^{(p)}$ of $K$ is divisible by $p$, then we have

$$
\left(K_{g}\right)_{1}=\left(K_{g}\right)_{1} K_{1}^{(p)} \varsubsetneqq\left(K_{g}\right)_{1} K_{2}^{(p)} \cong K_{2},
$$

because the odd part of the class group of $K_{g}$ is the direct product of the odd parts of the class groups of the quadratic subfields of $K_{g}$. If the $p$-rank of the $p$-class group of $K$ is larger than one for some odd prime $p$, then $h\left(K_{1}^{(p)}\right)$ is divisible by $p$ [37, Theorem 2] and therefore in this case $K_{2} \supsetneqq\left(K_{g}\right)_{1}$. For example, let $K=\mathbf{Q}(\sqrt{-3896})$. Then we have $\mathrm{Cl}(K) \cong C_{12} \times C_{3}, K_{g}=\mathrm{Q}(\sqrt{2}, \sqrt{-487})$, and $\mathrm{Cl}\left(K_{g}\right) \cong C_{42} \times C_{3}$. Therefore $K_{2} \supsetneqq K_{1}^{(3)}\left(K_{g}\right)_{1}=\left(K_{g}\right)_{1}$. Thus, for $K_{2}=\left(K_{g}\right)_{1}$, the $p$-class field tower must terminate with $K_{1}^{(p)}$ for all odd primes, in other words, the odd part of the class group of $K$ must be cyclic.

Problem 1 seems to be very difficult. Thus, it is reasonable to consider the following before treating Problem 1.

Problem 2. Characterize the imaginary quadratic number fields $K$ with

$$
K_{2}^{(2)}=\left(K_{g}\right)_{1}^{(2)} \neq K_{1}^{(2)}
$$

Put $\Lambda=\operatorname{Gal}\left(K_{2}^{(2)} / K\right)$. Then $\operatorname{Gal}\left(K_{2}^{(2)} / K_{1}^{(2)}\right)=\Lambda^{\prime}, \operatorname{Gal}\left(K_{2}^{(2)} / K_{g}\right)=\Phi(\Lambda)$, and $\operatorname{Gal}\left(K_{2}^{(2)} /\left(K_{g}\right)_{1}\right)=\Phi(\Lambda)^{\prime}$. Problem 2 consists in characterizing $K$ such that

$$
\Lambda^{\prime} \neq \Phi(\Lambda)^{\prime}=\left(\Lambda^{\prime \prime}=\right)\{1\}
$$

If $\mathrm{Cl}^{(2)}(K)$ is elementary abelian, then $K_{g}=K_{1}^{(2)}$ and therefore if moreover $h\left(K_{g}\right)$ is divisible by 2 , then $(* *)$ trivially holds. We can characterize such $K: \mathrm{Cl}^{(2)}(K)$ is elementary abelian if and only if $d$ does not have a factorization of the second kind (in the terminology of Rédei-Reichardt). F. Lemmermeyer [29] characterized $K$ for which $\Lambda$ is abelian. By known 
results on the 2-class field tower, if the 2-class group of $K_{1}^{(2)}$ is nontrivial and cyclic, (**) holds. (Refer to $\S 5$.) In fact, for $\Lambda=M C_{n}^{-}, M C_{n, m}^{+}, \Gamma_{m, t}^{1}$ or $\Gamma_{m, t}^{2},(* * *)$ holds. Therefore the remaining case is where $\mathrm{Cl}^{(2)}(K)$ is not elementary abelian and $\mathrm{Cl}^{(2)}\left(K_{1}^{(2)}\right)$ is noncyclic. The author knows no example of $K$ with $K_{2}^{(2)} \supsetneqq\left(K_{g}\right)_{1}^{(2)} \supsetneqq K_{1}^{(2)}$.

Finally, we note that there exist 2-groups $\Lambda$ satisfying $\Lambda^{\prime} \neq \Phi(\Lambda)^{\prime} \neq$ $\Lambda^{\prime \prime}=\{1\}$. The groups $64 \Gamma_{22} a_{1}, 64 \Gamma_{22} a_{2}, 64 \Gamma_{23} a_{1}, 64 \Gamma_{23} a_{2}, 64 \Gamma_{23} a_{3}$, and $64 \Gamma_{23} a_{4}$ are so. This is the complete list of the nonabelian 2-groups of orders $\leqq 64$ having nonabelian Frattini subgroup. (See [17].)

\section{REFERENCES}

1. S. Arno, The imaginary quadratic fields of class number 4, Acta Arith. 60 (1992), no. 4, 321-334; MR 93b:11144.

2. S. Arno, M. L. Robinson, and F. S. Wheeler, The imaginary quadratic fields of small odd class numbers, preprint, 1993.

3. J. Basmaji and I. Kiming, $A$ table of $A_{5}$-fields, On Artin's conjecture for odd 2-dimensional representations (G. Frey, ed.), Lecture Notes in Math., vol. 1585, Springer-Verlag, New York and Berlin, 1994, pp. 37-46, 122-141; MR 96e:11141.

4. E. Benjamin, Remarks concerning the 2-Hilbert class field of imaginary quadratic number fields, Bull Austral. Math. Soc. 48 (1993), no. 3, 379-383; MR 94m:11133; Corrigenda, ibid. 50 (1994), no. 2, 351-352.

5. E. Benjamin, F. Lemmermeyer, and C. Snyder, Imaginary quadratic fields $k$ with cyclic $\mathrm{Cl}_{2}\left(k^{1}\right)$, J. Number Theory 67 (1997), no. 2, 229-245.

6. R. Brauer, Beziehung zwischen Klassenzahl von Teilkörpern eines galoisschen Körpers, Math. Nachr. 4 (1951), no. 139, 158-174; MR 12, 593b; reprinted in Collected papers, vol. III, MIT Press, Cambridge, Mass.-London, 1980, pp. 497-513.

7. D. A. Buell, Small class number and extreme values of $L$-functions of quadratic fields, Math. Comp. 31 (1977), no. 139, 786-796; MR 55 \#12684.

8. R. Carter and P. Fong, The Sylow 2-subgroups of the finite classical groups, J. Algebra 1 (1964), no. 2, 139-151; MR 29 \#3548.

9. C. Castela, Nombre de classes d'idéaux d'une extension diédrale d'un corps de nombres, C. R. Acad. Sci. Paris Sér. A-B 287 (1978), no. 7, 483-486; MR 80c:12012.

10. H. Cohen and H. W. Lenstra, Jr., Heuristics on class groups of number fields, Number Theory, Noordwijkerhout, 1983 (H. Jager, ed.), Lecture Notes in Math., vol. 1068, Springer-Verlag, Berlin and New York, 1984, pp. 33-62; MR 85j:11144.

11. H. Cohn, A classical invitation to algebraic numbers and class fields, Universitext, Springer-Verlag, Berlin and New York, 1978; MR 80c:12001.

12. F. Diaz y Diaz, Tables minorant la racine $n$-ième du discriminant d'un corps de degré $n$, Publications Mathématiques d'Orsay 80, 6., Université de Paris-Sud, Département de Mathématique, Orsay, 1980; MR 82i:12007. 
13. H. J. Godwin, On quartic fields of signature one with small discriminant. II, Math. Comp. 42 (1984), no. 166, 707-711; MR 85i:11092a; Corrigendum, ibid. 43 (1984), no. 168,621 ; MR 85i:11092b.

14. - On totally complex quartic fields with small discriminant, Proc. Cambridge Philos. Soc. 53 (1957), 1-4; MR 18, 565c.

15. _ On relations between cubic and quartic fields, Quart. J. Math. Oxford (2) 13 (1962), 206-212; Corrigendum, ibid. (3) 26 (1975), no. 104, 511-512; MR 52 \#8078.

16. F. Hajir, Unramified elliptic units, thesis, MIT, 1993.

17. M. Hall, Jr. and J. K. Senior, The groups of order $2^{n}(n \leq 6)$, The Macmillan Co., New York, 1964; MR 29 \#5889.

18. F. Halter-Koch, Einheiten und Divisorenklassen in Galois'schen algebraischen Zahlkörpern mit Diedergruppe der Ordnung $2 l$ für eine ungerade Primzahl l, Acta Arith. 33 (1977), no. 4, 355-364; MR 56 \#11955.

19. F. Halter-Koch et N. Moser, Sur le nombre de classes de certaines extensions métacycliques sur $\mathbf{Q}$ ou sur un corps quadratiques imaginaires, J. Math. Soc. Japan 30 (1978), no. 2, 237-248; MR 58 \#5587.

20. H. Hayashi, On elliptic units and class number of a certain dihedral extension of degree 2l, Acta Arith. 45 (1985), no. 1, 35-45; MR 86m:11081.

21. C. S. Herz, Construction of class fields, Seminar on Complex Multiplication, Chap. VII, Lecture Notes in Math., vol. 21, Springer-Verlag, Berlin and New York, 1966.

22. B. Huppert, Endliche Gruppen I, Die Grundlehren der math. Wiss., Bd. 134, SpringerVerlag, Berlin and New York, 1967; MR 37 \#302.

23. A. Jehanne, Sur les extensions de $\mathbf{Q}$ à groupe de Galois $S_{4}$ et $\widetilde{S_{4}}$, Acta Arith. 70 (1995), no. 3, 259-276; MR 95m:11127.

24. G. Kientega and P. Barrucand, On quartic fields with symmetric group, Number theory (R. A. Mollin, ed.), de Gruyter, Berlin, 1990, pp. 287-297; MR 92e:11113.

25. H. Kisilevsky, Number fields with class number congruent to 4 mod 8 and Hilbert's Theorem 94, J. Number Theory 8 (1976), no. 3, 271-279; MR 54 \#5188.

26. T. Kondo, Algebraic number fields with the discriminant equal to that of a quadratic number field, J. Math. Soc. Japan 47 (1995), no. 1, 31-36; MR 95h:11121.

27. S. Kuroda, Über die Klassenzahlen algebraischer Zahlkörper, Nagoya Math. J. 1 (1950), 1-10; MR 12, 593a.

28. F. Lemmermeyer, Kuroda's class number formula, Acta Arith. 66 (1994), no. 3, 245-260; MR 95f:11090.

29. — On 2-class field towers of imaginary quadratic number fields, J. Théor. Nombres Bordeaux 6 (1994), no. 2, 261-272; MR 96k:11136.

30. - On unramified quaternion extension of imaginary quadratic number fields, J. Théor. Nombres Bordeaux 9 (1997), no. 1, 51-68.

31. - On 2-Class field towers of some imaginary quadratic number fields, Abh. Math. Sem. Univ. Hamburg 67 (1997), 205-214. 
32. Private communication, 1996.

33. J. Martinet, Corps de nombres de classes 1, Séminaire de Théorie des Nombres 1977-1978, Exp. No. 12, CNRS, Talence, 1978; MR 80k:12009.

34. __ Petits discriminants des corps de nombres, Number theory days, 1980 (Exeter, 1980), London Math. Soc. Lecture Note Ser. 56, Cambridge Univ. Press, Cambridge, New York, 1982, pp. 151-193; MR 84g:12009.

35. J. M. Masley, Class numbers of real cyclic number fields with small conductor, Compositio Math. 37 (1978), no. 3, 297-319; MR 80e:12005.

36. N. Moser, Unités et nombre de classes d'une extension galoisienne diédrale de $\mathbf{Q}$, Abh. Math. Sem. Univ. Hamburg 48 (1979), 54-75; MR 81h:12009.

37. A. Nomura, On the existence of unramified p-extensions, Osaka J. Math. 28 (1991), no. 1, 55-62; MR 92e:11115.

38. A. M. Odlyzko, Discriminant bounds, (unpublished tables), Nov. 29th 1976.

39. _ـ Bounds for discriminants and related estimates for class numbers, regulators and zeros of zeta functions : a survey of recent results, Sém. Théor. Nombres Bordeaux (2) 2 (1990), no. 1, 119-141; MR 91i:11154.

40. J. Oesterlé, Nombres de classes de corps quadratiques imaginaires, Sém. Bourbaki 1983-1984, Exp. 631, 14pp; MR 86k:11064.

41. M. Olivier, Corps sextique primitifs, Ann. Inst. Fourier (Grenoble) 40 (1990), no. 4, 757-767; MR 92a:11123.

42. T. W. Sag and J. W. Wamsley, Minimal presentations for groups of order $2^{n}, n \leqq 6$, J. Austral. Math. Soc. 15 (1973), 461-469; MR 49 \#406.

43. R. Schoof, Private communication, 1996.

44. A. Schwarz, M. Pohst and F. Diaz y Diaz, A table of quintic number fields, Math. Comp. 63 (1994), no. 207, 361-374; MR 94i:11108.

45. J.-P. Serre, Modular forms of weight one and Galois representations, Algebraic number fields: $L$-functions and Galois properties (Proc. Sympos., Univ. Durham, Durham, 1975) (A. Fröhlich, ed.), Academic Press, London, 1977, pp. 193-268; MR 56 \#8497; repreinted in Collected papres, vol. III, Springer-Verlag, New York and Berlin, 1986, pp. 292-367.

46. _ Topics in Galois theory, Research Notes in Math., vol. 1, Jones and Bartlett Publishers, Boston, MA, 1992; MR 94d:12006.

47. M. W. Short, The primitive soluble permutation groups of degree less than 256, Lecture Notes in Math., vol. 1519, Springer-Verlag, Berlin and New York, 1992; MR 93g:20006.

48. A. G. Stephens and H. C. Williams, Computation of real quadratic fields with class number one, Math. Comp. 51 (1988), no. 184, 809-824; MR 90b:11106.

49. O. Taussky, A remark on the class field tower, J. London Math. Soc. 12 (1937), 82-85.

50. F. van der Linden, Class number computations of real abelian number fields, Math. Comp. 39 (1982), no. 160, 693-707; MR 84e:12005. 
51. H. Wada, On the class number and the unit group of certain algebraic number fields, J. Fac. Sci. Univ. Tokyo Sect. IA 13 (1966), 201-209; MR 35 \#5414.

52. C. Wagner, Class number 5, 6 and 7, Math. Comp. 65 (1996), no. 214, 785-800; MR 96g:11135.

53. L. C. Washington, Introduction to Cyclotomic Fields, Graduate Text in Math., vol. 83, Springer-Verlag, Berlin and New York, 1982; MR 85g:11101.

54. Y. Yamamoto, Divisibility by 16 of class numbers of quadratic fields whose 2-class groups are cyclic, Osaka J. Math. 21 (1984), no. 1, 1-22; MR 85g:11092.

55. K. Yamamura, On unramified Galois extensions of real quadratic number fields, Osaka J. Math. 23 (1986), no. 2, 471-486; MR 88a:11112.

56.

Some analogue of Hilbert's irreducibility theorem and the distribution of algebraic number fields, J. Fac. Sci. Univ. Tokyo Sect. IA 38 (1991), no. 1, 99-135; MR 92e:11132.

57. $\longrightarrow$ The determination of the imaginary abelian number fields with class number one, Math. Comp. 62 (1994), no. 206, 899-921; MR 94g:11096.

58. — The maximal unramified extensions of the imaginary quadratic number fields with class number two, J. Number Theory 60 (1996), no. 2, 42-50; MR 97g:11119.

59. - Determination of the non-CM imaginary normal octic number fields with class number one, submitted for publication.

60. $\longrightarrow$ Real quadratic number fields with class number one having an unramified $A_{n}$-extension, in preparation.

61. K. Yamazaki, Computations of Galois groups, Proc. Symp. Group theory and its application (T. Kondo, ed.), 1981, pp. 9-57. (Japanese)

Ken YAMAMURA

Department of Mathematics

National Defence Academy

Hashirimizu Yokosuka 239

JAPAN

e-mail : yamamura@cc.nda.ac.jp 